\title{
HOMOGENEOUS KOBAYASHI-HYPERBOLIC MANIFOLDS WITH HIGH-DIMENSIONAL GROUP OF HOLOMORPHIC AUTOMORPHISMS
}

\author{
ALEXANDER ISAEV
}

\begin{abstract}
We determine all connected homogeneous Kobayashi-hyperbolic manifolds of dimension $n \geq 2$ whose holomorphic automorphism group has dimension $n^{2}-2$. This result complements an existing classification for automorphism group dimension $n^{2}-1$ and greater obtained without the homogeneity assumption.
\end{abstract}

\section{INTRODUCTION}

Let $M$ be a connected complex manifold of dimension $n \geq 2$. It is called Kobayashi-hyperbolic if the Kobayashi pseudodistance $K_{M}$ on $M$ is in fact a distance, i.e., for $p, q \in M$ the identity $K_{M}(p, q)=0$ implies $p=q$. For example, any bounded domain in complex space $\mathbb{C}^{n}$ is Kobayashi-hyperbolic. Such manifolds are of substantial interest in complex analysis and geometry as they possess many attractive properties (see monographs [K1], [K2] for details). In particular, if $M$ is Kobayashi-hyperbolic, the group $\operatorname{Aut}(M)$ of its holomorphic automorphisms is a (real) Lie group in the compact-open topology (see [K1, Chapter V, Theorem 2.1]). One way to prove this is by observing that the action of $\operatorname{Aut}(M)$ on $M$ is proper, which implies that $\operatorname{Aut}(M)$ is locally compact hence a Lie transformation group (see [I5] for details).

Let $d(M):=\operatorname{dim} \operatorname{Aut}(M)$. It is a classical fact that $d(M) \leq n^{2}+2 n$ and the equality holds if and only if $M$ is biholomorphic to the unit ball $B^{n} \subset \mathbb{C}^{n}$ (see [K1, Chapter V, Theorem 2.6]). In papers [I1], [I2], [I4], [IK] we classified all Kobayashihyperbolic manifolds with $n^{2}-1 \leq d(M)<n^{2}+2 n$ (see also [I3], [I5] for alternative expositions of these results). In particular, it turned out that no manifolds satisfy $n^{2}+3 \leq d(M)<n^{2}+2 n$, i.e., that the possible values of $d(M)$ contain a lacuna located between $n^{2}+2$ and the maximal dimension $n^{2}+2 n$. We note that the lacunary behavior of $d(M)$ is analogous to that of the dimension of the isometry group of a Riemannian manifold (see, e.g., [I5] for a brief survey).

Our classification has turned out to be rather useful in applications (see, e.g., [GIL], [V]), and it would be desirable to extend it beyond the case $d(M)=n^{2}-1$. However, the value $n^{2}-2$ is critical in the sense that one cannot hope to obtain a full explicit description of Kobayashi-hyperbolic manifolds for $d(M)=n^{2}-2$ and all $n \geq 2$. Indeed, a generic Reinhardt domain in $\mathbb{C}^{2}$ has a 2 -dimensional automorphism group, so no reasonable classification exists for $n=2$ (see [I3, pp. 67] for a precise argument). Furthermore, producing an explicit classification for $n \geq 3$ appears to be out of reach either as the amount of work required to deal even with the easier case $d(M)=n^{2}-1$ is already rather substantial (see [I1], [I4]).

At the same time, some hope remains in the situation when $M$ is homogeneous, i.e., when the action of $\operatorname{Aut}(M)$ on $M$ is transitive. Such manifolds are of general interest, and we focus on them in this paper. Specifically, in the following theorem

Mathematics Subject Classification: 32Q45, 32M05, 32M10.

Keywords: Kobayashi-hyperbolic manifolds, homogeneous complex manifolds, the group of holomorphic automorphisms. 
we obtain a description of homogeneous Kobayashi-hyperbolic manifolds satisfying $d(M)=n^{2}-2$. It is remarkably easy to state (although not so easy to prove):

THEOREM 1.1. Let $M$ be a homogeneous Kobayashi-hyperbolic manifold with $d(M)=n^{2}-2$. Then $M$ is biholomorphic either to $B^{2} \times B^{1} \times B^{1}$ (here $\left.n=4\right)$ or to $B^{3} \times B^{2}($ here $n=5)$.

Combined with the classical fact for $d(M)=n^{2}+2 n$ mentioned above and [I3, Theorem 2.2], this result yields:

THEOREM 1.2. Let $M$ be a homogeneous Kobayashi-hyperbolic manifold satisfying $n^{2}-2 \leq d(M) \leq n^{2}+2 n$. Then $M$ is biholomorphic either to a suitable product of balls or to a suitable symmetric bounded domain of type (IV). Specifically, the following products of balls are possible:

(i) $B^{n}\left(\right.$ here $\left.d(M)=n^{2}+2 n\right)$,

(ii) $B^{n-1} \times B^{1}$ (here $\left.d(M)=n^{2}+2\right)$,

(iii) $B^{1} \times B^{1} \times B^{1}$ (here $n=3, d(M)=9=n^{2}$ ),

(iv) $B^{2} \times B^{2}$ (here $n=4, d(M)=16=n^{2}$ ),

(v) $B^{2} \times B^{1} \times B^{1}$ (here $\left.n=4, d(M)=14=n^{2}-2\right)$,

(vi) $B^{3} \times B^{2}$ (here $n=5, d(M)=23=n^{2}-2$ ),

and the following symmetric bounded domains of type (IV) (written in tube form) are possible:

(vii) the domain of type $\left(\mathrm{IV}_{3}\right)$

$$
\begin{aligned}
& T_{3}:=\left\{\left(z_{1}, z_{2}, z_{3}\right) \in \mathbb{C}^{3}:\left(\operatorname{Im} z_{1}\right)^{2}-\left(\operatorname{Im} z_{2}\right)^{2}-\left(\operatorname{Im} z_{3}\right)^{2}>0, \operatorname{Im} z_{1}>0\right\} \\
& \text { (here } \left.n=3, d(M)=10=n^{2}+1\right),
\end{aligned}
$$

(viii) the domain of type $\left(\mathrm{IV}_{4}\right)$

$$
\begin{aligned}
& T_{4}:=\left\{\left(z_{1}, z_{2}, z_{3}, z_{4}\right) \in \mathbb{C}^{4}:\left(\operatorname{Im} z_{1}\right)^{2}-\left(\operatorname{Im} z_{2}\right)^{2}-\right. \\
& \left.\quad\left(\operatorname{Im} z_{3}\right)^{2}-\left(\operatorname{Im} z_{4}\right)^{2}>0, \operatorname{Im} z_{1}>0\right\}
\end{aligned}
$$

The proof of Theorem 1.1 is given in Section 3 and is based on reduction to the case of the so-called Siegel domains of the second kind introduced by I. PyatetskiiShapiro at the end of the 1950s in relation to problems in the theory of automorphic functions (see Section 2 for the definition and some properties of such domains). Indeed, in the seminal work [VGP-S] it was shown that every homogeneous bounded domain in $\mathbb{C}^{n}$ is biholomorphic to an affinely homogeneous Siegel domain of the second kind. Furthermore, in [N] this result was extended to arbitrary homogeneous Kobayashi-hyperbolic manifolds, which solved a problem posed in [K1] (see Problem 8 on p. 127 therein). The proof of Theorem 1.1 then follows by analyzing the (graded) Lie algebra of the automorphism group of a Siegel domain of the second kind, which was described in [KMO], [S, Chapter V, §1-2]. The analysis of this Lie algebra also yields additional facts (included in the appendix) that can be utilized for extending the classifications in Theorems 1.1, 1.2 beyond the critical automorphism group dimension $n^{2}-2$. Our arguments show that the formulas for the graded components of the algebra provided in [KMO], [S] can be quite useful in applications despite the fact that explicit calculations involving these formulas are rarely seen in the literature.

Acknowledgement. This work is supported by the Australian Research Council. 


\section{Preliminaries on Convex Cones and Siegel Domains of THE SECOND KIND}

In this section we define Siegel domains of the second kind and collect their properties as required for our proof of Theorem 1.1 in the next section.

First of all, an open subset $\Omega \subset \mathbb{R}^{k}$ is called an open convex cone if it is closed with respect to taking linear combinations of its elements with positive coefficients. Such a cone $\Omega$ is called (linearly) homogeneous if the group

$$
G(\Omega):=\left\{A \in \mathrm{GL}_{k}(\mathbb{R}): A \Omega=\Omega\right\}
$$

of linear automorphisms of $\Omega$ acts transitively on it. Clearly, $G(\Omega)$ is a closed subgroup of $\mathrm{GL}_{k}(\mathbb{R})$, and we denote by $\mathfrak{g}(\Omega) \subset \mathfrak{g l}_{k}(\mathbb{R})$ its Lie algebra.

We will be interested in open convex cones not containing entire lines. For such cones the dimension of $\mathfrak{g}(\Omega)$ admits a useful estimate.

Lemma 2.1. Let $\Omega \subset \mathbb{R}^{k}$ be an open convex cone not containing a line. Then

$$
\operatorname{dim} \mathfrak{g}(\Omega) \leq \frac{k^{2}}{2}-\frac{k}{2}+1
$$

Proof. Fix a point $\mathbf{x} \in \Omega$ and consider its isotropy subgroup $G_{\mathbf{x}}(\Omega) \subset G(\Omega)$. This subgroup is compact since it leaves invariant the bounded open set $\Omega \cap(\mathbf{x}-\Omega)$. Therefore, changing variables in $\mathbb{R}^{k}$ if necessary, we can assume that $G_{\mathbf{x}}(\Omega)$ lies in the orthogonal group $\mathrm{O}_{k}(\mathbb{R})$. The group $\mathrm{O}_{k}(\mathbb{R})$ acts transitively on the sphere of radius $\|\mathbf{x}\|$ in $\mathbb{R}^{k}$, and the isotropy subgroup $I_{\mathbf{x}}$ of $\mathbf{x}$ under the $\mathrm{O}_{k}(\mathbb{R})$-action is isomorphic to $\mathrm{O}_{k-1}(\mathbb{R})$. Since $G_{\mathbf{x}}(\Omega) \subset I_{\mathbf{x}}$, we have

$$
\operatorname{dim} G_{\mathbf{x}}(\Omega) \leq \operatorname{dim} I_{\mathbf{x}}=\frac{k^{2}}{2}-\frac{3 k}{2}+1,
$$

which implies inequality (2.1).

Next, let

$$
H: \mathbb{C}^{m} \times \mathbb{C}^{m} \rightarrow \mathbb{C}^{k}
$$

be a Hermitian form on $\mathbb{C}^{m}$ with values in $\mathbb{C}^{k}$, where we assume that $H\left(w, w^{\prime}\right)$ is linear in $w^{\prime}$ and anti-linear in $w$. For an open convex cone $\Omega \subset \mathbb{R}^{k}$, the form $H$ is called $\Omega$-Hermitian if $H(w, w) \in \bar{\Omega} \backslash\{0\}$ for all non-zero $w \in \mathbb{C}^{m}$. Observe that if $\Omega$ contains no lines and $H$ is $\Omega$-Hermitian, then there exists a positive-definite linear combination of the components of $H$.

Now, a Siegel domain of the second kind in $\mathbb{C}^{n}$ is an unbounded domain of the form

$$
S(\Omega, H):=\left\{(z, w) \in \mathbb{C}^{k} \times \mathbb{C}^{n-k}: \operatorname{Im} z-H(w, w) \in \Omega\right\}
$$

for some $1 \leq k \leq n$, some open convex cone $\Omega \subset \mathbb{R}^{k}$ not containing a line, and some $\Omega$-Hermitian form $H$ on $\mathbb{C}^{n-k}$. For $k=n$ we have $H=0$, so in this case $S(\Omega, H)$ is the tube domain

$$
\left\{z \in \mathbb{C}^{n}: \operatorname{Im} z \in \Omega\right\} .
$$

Such tube domains are often called Siegel domains of the first kind. At the other extreme, when $k=1$, the domain $S(\Omega, H)$ is linearly equivalent to

$$
\left\{(z, w) \in \mathbb{C} \times \mathbb{C}^{n-1}: \operatorname{Im} z-\|w\|^{2}>0\right\},
$$

which is an unbounded realization of the unit ball $B^{n}$ (see [R, p. 31]). In fact, any Siegel domain of the second kind is biholomorphic to a bounded domain (see [P-S, pp. 23-24]), hence is Kobayashi-hyperbolic.

Next, the holomorphic affine automorphisms of Siegel domains of the second kind are described as follows (see [P-S, pp. 25-26]): 
THEOREM 2.2. Any holomorphic affine automorphism of $S(\Omega, H)$ has the form

$$
\begin{aligned}
z & \mapsto A z+a+2 i H(b, B w)+i H(b, b), \\
w & \mapsto B w+b,
\end{aligned}
$$

with $a \in \mathbb{R}^{k}, b \in \mathbb{C}^{n-k}, A \in G(\Omega), B \in \mathrm{GL}_{n-k}(\mathbb{C})$, where

$$
A H\left(w, w^{\prime}\right)=H\left(B w, B w^{\prime}\right)
$$

for all $w, w^{\prime} \in \mathbb{C}^{n-k}$.

A domain $S(\Omega, H)$ is called affinely homogeneous if the group $\operatorname{Aff}(S(\Omega, H))$ of its holomorphic affine automorphisms acts on $S(\Omega, H)$ transitively. Denote by $G(\Omega, H)$ the subgroup of $G(\Omega)$ that consists of all transformations $A \in G(\Omega)$ as in Theorem 2.2, namely, of all elements $A \in G(\Omega)$ for which there exists $B \in \mathrm{GL}_{n-k}(\mathbb{C})$ such that (2.2) holds. By [D, Lemma 1.1], the subgroup $G(\Omega, H)$ is closed in $G(\Omega)$. It is easy to deduce from Theorem 2.2 that if $S(\Omega, H)$ is affinely homogeneous, the action of $G(\Omega, H)$ (hence that of its identity component $\left.G(\Omega, H)^{\circ}\right)$ is transitive on $\Omega$ (see, e.g., [KMO, proof of Theorem 8]), so the cone $\Omega$ is homogeneous. Conversely, if $G(\Omega, H)$ acts on $\Omega$ transitively, the domain $S(\Omega, H)$ is affinely homogeneous.

As shown in [VGP-S], [N], every homogeneous Kobayashi-hyperbolic manifold is biholomorphic to an affinely homogeneous Siegel domain of the second kind. Such a realization is unique up to affine transformations; in general, if two Siegel domains of the second kind are biholomorphic to each other, they are also equivalent by means of a linear transformation of special form (see [KMO, Theorem 11]). The result of [VGP-S], [N] is the basis of our proof of Theorem 1.1 in the next section.

In addition, our proof relies on a description of the Lie algebra of the group $\operatorname{Aut}(S(\Omega, H))$ of an arbitrary Siegel domain of the second kind $S(\Omega, H)$. This algebra is isomorphic to the (real) Lie algebra of complete holomorphic vector fields on $S(\Omega, H)$, which we denote by $\mathfrak{g}(S(\Omega, H))$ or, when there is no fear of confusion, simply by $\mathfrak{g}$. This algebra has been extensively studied. In particular, we have (see [KMO, Theorems 4 and 5]):

THEOREM 2.3. The algebra $\mathfrak{g}=\mathfrak{g}(S(\Omega, H))$ admits a grading

$$
\mathfrak{g}=\mathfrak{g}_{-1} \oplus \mathfrak{g}_{-1 / 2} \oplus \mathfrak{g}_{0} \oplus \mathfrak{g}_{1 / 2} \oplus \mathfrak{g}_{1},
$$

with $\mathfrak{g}_{\nu}$ being the eigenspace with eigenvalue $\nu$ of ad $\partial$, where $\partial:=z \cdot \frac{\partial}{\partial z}+\frac{1}{2} w \cdot \frac{\partial}{\partial w}$. Here

$$
\begin{array}{ll}
\mathfrak{g}_{-1}=\left\{a \cdot \frac{\partial}{\partial z}: a \in \mathbb{R}^{k}\right\}, & \operatorname{dim} \mathfrak{g}_{-1}=k, \\
\mathfrak{g}_{-1 / 2}=\left\{2 i H(b, w) \cdot \frac{\partial}{\partial z}+b \cdot \frac{\partial}{\partial w}: b \in \mathbb{C}^{n-k}\right\}, & \operatorname{dim} \mathfrak{g}_{-1 / 2}=2(n-k),
\end{array}
$$

and $\mathfrak{g}_{0}$ consists of all vector fields of the form

$$
(A z) \cdot \frac{\partial}{\partial z}+(B w) \cdot \frac{\partial}{\partial w}
$$

with $A \in \mathfrak{g}(\Omega), B \in \mathfrak{g l}_{n-k}(\mathbb{C})$ and

$$
A H\left(w, w^{\prime}\right)=H\left(B w, w^{\prime}\right)+H\left(w, B w^{\prime}\right)
$$

for all $w, w^{\prime} \in \mathbb{C}^{n-k}$. Furthermore, one has

$$
\operatorname{dim} \mathfrak{g}_{1 / 2} \leq 2(n-k), \quad \operatorname{dim} \mathfrak{g}_{1} \leq k .
$$

It is then clear that the matrices $A$ that appear in (2.3) form the Lie algebra of $G(\Omega, H)$ and that $\mathfrak{g}_{-1} \oplus \mathfrak{g}_{-1 / 2} \oplus \mathfrak{g}_{0}$ is isomorphic to the Lie algebra of the group $\operatorname{Aff}(S(\Omega, H))$ (compare conditions (2.2) and (2.4)). 
Following [S], for a pair of matrices $A, B$ satisfying (2.4) we say that $B$ is associated to $A$ (with respect to $H$ ). Let $\mathcal{L}$ be the (real) subspace of $\mathfrak{g l}_{n-k}(\mathbb{C})$ of all matrices associated to the zero matrix in $\mathfrak{g}(\Omega)$, i.e., matrices skew-Hermitian with respect to each component of $H$. Set $s:=\operatorname{dim} \mathcal{L}$. Then we have

$$
\operatorname{dim} \mathfrak{g}_{0} \leq s+\operatorname{dim} \mathfrak{g}(\Omega) .
$$

By Theorem 2.3 and inequality (2.6) one obtains

$$
d(S(\Omega, H)) \leq k+2(n-k)+s+\operatorname{dim} \mathfrak{g}(\Omega)+\operatorname{dim} \mathfrak{g}_{1 / 2}+\operatorname{dim} \mathfrak{g}_{1},
$$

which, combined with (2.5) leads to

$$
d(S(\Omega, H)) \leq 2 k+4(n-k)+s+\operatorname{dim} \mathfrak{g}(\Omega) .
$$

Further, since there exists a positive-definite linear combination $\mathbf{H}$ of the components of the Hermitian form $H$, the subspace $\mathcal{L}$ lies in the Lie algebra of matrices skew-Hermitian with respect to $\mathbf{H}$, thus

$$
s \leq(n-k)^{2} .
$$

By (2.9), inequality (2.8) yields

$$
d(S(\Omega, H)) \leq 2 k+4(n-k)+(n-k)^{2}+\operatorname{dim} \mathfrak{g}(\Omega) .
$$

Combining (2.10) with (2.1), we deduce the following useful upper bound:

$$
d(S(\Omega, H)) \leq \frac{3 k^{2}}{2}-k\left(2 n+\frac{5}{2}\right)+n^{2}+4 n+1 .
$$

Next, by [S, Chapter V, Proposition 2.1] the component $\mathfrak{g}_{1 / 2}$ of the Lie algebra $\mathfrak{g}=\mathfrak{g}(S(\Omega, H))$ is described as follows:

THEOREM 2.4. The subspace $\mathfrak{g}_{1 / 2}$ consists of all vector fields of the form

$$
2 i H(\Phi(\bar{z}), w) \cdot \frac{\partial}{\partial z}+(\Phi(z)+c(w, w)) \cdot \frac{\partial}{\partial w},
$$

where $\Phi: \mathbb{C}^{k} \rightarrow \mathbb{C}^{n-k}$ is a $\mathbb{C}$-linear map such that for every $\mathbf{w} \in \mathbb{C}^{n-k}$ one has

$$
\Phi_{\mathbf{w}}:=\left[x \mapsto \operatorname{Im} H(\mathbf{w}, \Phi(x)), x \in \mathbb{R}^{k}\right] \in \mathfrak{g}(\Omega),
$$

and $c: \mathbb{C}^{n-k} \times \mathbb{C}^{n-k} \rightarrow \mathbb{C}^{n-k}$ is a symmetric $\mathbb{C}$-bilinear form on $\mathbb{C}^{n-k}$ with values in $\mathbb{C}^{n-k}$ satisfying the condition

$$
H\left(w, c\left(w^{\prime}, w^{\prime}\right)\right)=2 i H\left(\Phi\left(H\left(w^{\prime}, w\right)\right), w^{\prime}\right)
$$

for all $w, w^{\prime} \in \mathbb{C}^{n-k}$.

Further, by [S, Chapter V, Proposition 2.2], the component $\mathfrak{g}_{1}$ of $\mathfrak{g}=\mathfrak{g}(S(\Omega, H))$ admits the following description:

THEOREM 2.5. The subspace $\mathfrak{g}_{1}$ consists of all vector fields of the form

$$
a(z, z) \cdot \frac{\partial}{\partial z}+b(z, w) \cdot \frac{\partial}{\partial w}
$$

where $a: \mathbb{R}^{k} \times \mathbb{R}^{k} \rightarrow \mathbb{R}^{k}$ is a symmetric $\mathbb{R}$-bilinear form on $\mathbb{R}^{k}$ with values in $\mathbb{R}^{k}$ (which we extend to a symmetric $\mathbb{C}$-bilinear form on $\mathbb{C}^{k}$ with values in $\mathbb{C}^{k}$ ) such that for every $\mathbf{x} \in \mathbb{R}^{k}$ one has

$$
A_{\mathbf{x}}:=\left[x \mapsto a(\mathbf{x}, x), x \in \mathbb{R}^{k}\right] \in \mathfrak{g}(\Omega),
$$

and $b: \mathbb{C}^{k} \times \mathbb{C}^{n-k} \rightarrow \mathbb{C}^{n-k}$ is a $\mathbb{C}$-bilinear map such that, if for $\mathbf{x} \in \mathbb{R}^{k}$ one sets

$$
B_{\mathbf{x}}:=\left[w \mapsto \frac{1}{2} b(\mathbf{x}, w), w \in \mathbb{C}^{n-k}\right],
$$

the following conditions are satisfied: 
(i) $B_{\mathbf{x}}$ is associated to $A_{\mathbf{x}}$ and $\operatorname{Im} \operatorname{tr} B_{\mathbf{x}}=0$ for all $\mathbf{x} \in \mathbb{R}^{k}$,

(ii) for every pair $\mathbf{w}, \mathbf{w}^{\prime} \in \mathbb{C}^{n-k}$ one has

$$
B_{\mathbf{w}, \mathbf{w}^{\prime}}:=\left[x \mapsto \operatorname{Im} H\left(\mathbf{w}^{\prime}, b(x, \mathbf{w})\right), x \in \mathbb{R}^{k}\right] \in \mathfrak{g}(\Omega),
$$

(iii) $H\left(w, b\left(H\left(w^{\prime}, w^{\prime \prime}\right), w^{\prime \prime}\right)\right)=H\left(b\left(H\left(w^{\prime \prime}, w\right), w^{\prime}\right), w^{\prime \prime}\right)$ for all $w, w^{\prime}, w^{\prime \prime} \in \mathbb{C}^{n-k}$.

Next, let us recall the well-known classification, up to linear equivalence, of homogeneous convex cones not containing lines in dimensions $k=2,3,4$ (see, e.g., [KT, pp. 38-41]), which will be also required for our proof of Theorem 1.1:

$k=2: \quad \Omega_{1}:=\left\{\left(x_{1}, x_{2}\right) \in \mathbb{R}^{2}: x_{1}>0, x_{2}>0\right\}$, where the algebra $\mathfrak{g}\left(\Omega_{1}\right)$ consists of all diagonal matrices, hence $\operatorname{dim} \mathfrak{g}\left(\Omega_{1}\right)=2$,

$k=3: \quad$ (i) $\Omega_{2}:=\left\{\left(x_{1}, x_{2}, x_{3}\right) \in \mathbb{R}^{3}: x_{1}>0, x_{2}>0, x_{3}>0\right\}$, where the algebra $\mathfrak{g}\left(\Omega_{2}\right)$ consists of all diagonal matrices, hence $\operatorname{dim} \mathfrak{g}\left(\Omega_{2}\right)=3$,

(ii) $\Omega_{3}:=\left\{\left(x_{1}, x_{2}, x_{3}\right) \in \mathbb{R}^{3}: x_{1}^{2}-x_{2}^{2}-x_{3}^{2}>0, x_{1}>0\right\}$, where one has $\mathfrak{g}\left(\Omega_{3}\right)=\mathfrak{c}\left(\mathfrak{g l}_{3}(\mathbb{R})\right) \oplus \mathfrak{o}_{1,2}$, hence $\operatorname{dim} \mathfrak{g}\left(\Omega_{3}\right)=4$; here for any Lie algebra $\mathfrak{h}$ we denote by $\mathfrak{c}(\mathfrak{h})$ its center,

$k=4: \quad$ (i) $\Omega_{4}:=\left\{\left(x_{1}, x_{2}, x_{3}, x_{4}\right) \in \mathbb{R}^{4}: x_{1}>0, x_{2}>0, x_{3}>0, x_{4}>0\right\}$, where the algebra $\mathfrak{g}\left(\Omega_{4}\right)$ consists of all diagonal matrices, hence we have $\operatorname{dim} \mathfrak{g}\left(\Omega_{4}\right)=4$,

(ii) $\Omega_{5}:=\left\{\left(x_{1}, x_{2}, x_{3}, x_{4}\right) \in \mathbb{R}^{4}: x_{1}^{2}-x_{2}^{2}-x_{3}^{2}>0, x_{1}>0, x_{4}>0\right\}$, where the algebra $\mathfrak{g}\left(\Omega_{5}\right)=\left(\mathfrak{c}\left(\mathfrak{g l}_{3}(\mathbb{R})\right) \oplus \mathfrak{o}_{1,2}\right) \oplus \mathbb{R}$ consists of block-diagonal matrices with blocks of sizes $3 \times 3$ and $1 \times 1$ corresponding to the two summands, hence $\operatorname{dim} \mathfrak{g}\left(\Omega_{5}\right)=5$,

(iii) $\Omega_{6}:=\left\{\left(x_{1}, x_{2}, x_{3}, x_{4}\right) \in \mathbb{R}^{4}: x_{1}^{2}-x_{2}^{2}-x_{3}^{2}-x_{4}^{2}>0, x_{1}>0\right\}$, where $\mathfrak{g}\left(\Omega_{6}\right)=\mathfrak{c}\left(\mathfrak{g l}_{4}(\mathbb{R})\right) \oplus \mathfrak{o}_{1,3}$, hence $\operatorname{dim} \mathfrak{g}\left(\Omega_{6}\right)=7$.

In $[\mathrm{C}]$, É. Cartan found all homogeneous bounded domains in $\mathbb{C}^{2}$ and $\mathbb{C}^{3}$. We conclude this section with a short proof of Cartan's theorem (extended to the case of Kobayashi-hyperbolic manifolds) based on Siegel domains of the second kind.

\section{THEOREM 2.6.}

(1) Every homogeneous Kobayashi-hyperbolic manifold of dimension 2 is biholomorphic to one of

(i) $B^{2}$,

(ii) $B^{1} \times B^{1}$.

(2) Every homogeneous Kobayashi-hyperbolic manifold of dimension 3 is biholomorphic to one of

(i) $B^{3}$,

(ii) $B^{2} \times B^{1}$,

(iii) $B^{1} \times B^{1} \times B^{1}$,

(iv) the tube domain $T_{3}$ defined in (1.1).

Proof. Let $M$ be a homogeneous Kobayashi-hyperbolic manifold of dimension $n$. By [VGP-S], [N], the manifold $M$ is biholomorphic to a Siegel domain of the second kind $S(\Omega, H)$. If $k=1$, then $S(\Omega, H)$ is biholomorphic to $B^{n}$, so we assume that $k \geq 2$.

If $n=2$, then $k=2$, hence after a linear change of variables $S(\Omega, H)$ becomes

$$
\left\{z \in \mathbb{C}^{2}: \operatorname{Im} z \in \Omega_{1}\right\}
$$

and therefore is biholomorphic to $B^{1} \times B^{1}$. This establishes Part (1). 
Assume that $n=3$ and suppose first that $k=3$. Then after a linear change of variables $S(\Omega, H)$ turns into one of the domains

$$
\begin{aligned}
& \left\{z \in \mathbb{C}^{3}: \operatorname{Im} z \in \Omega_{2}\right\}, \\
& \left\{z \in \mathbb{C}^{3}: \operatorname{Im} z \in \Omega_{3}\right\}
\end{aligned}
$$

and therefore is biholomorphic to either $B^{1} \times B^{1} \times B^{1}$ or the tube domain $T_{3}$.

Let now $k=2$. In this case, after a linear change of variables $S(\Omega, H)$ becomes

$$
D:=\left\{(z, w) \in \mathbb{C}^{2} \times \mathbb{C}: \operatorname{Im} z-v|w|^{2} \in \Omega_{1}\right\},
$$

where $v=\left(v_{1}, v_{2}\right)$ is a non-zero vector in $\mathbb{R}^{2}$ with non-negative components. Let us compute the group $G\left(\Omega_{1}, v|w|^{2}\right)$. It consists of all non-degenerate diagonal matrices

$$
\left(\begin{array}{cc}
\lambda_{1} & 0 \\
0 & \lambda_{2}
\end{array}\right),\left(\begin{array}{cc}
0 & \mu_{1} \\
\mu_{2} & 0
\end{array}\right)
$$

such that $\lambda_{1}>0, \lambda_{2}>0, \lambda_{1} v_{1}=\rho v_{1}, \lambda_{2} v_{2}=\rho v_{2}$ and $\mu_{1}>0, \mu_{2}>0, \mu_{1} v_{2}=\eta v_{1}$, $\mu_{2} v_{1}=\eta v_{2}$ for some $\rho, \eta>0$. Hence if $v_{1} \neq 0, v_{2} \neq 0$ we have

$$
G\left(\Omega_{1}, v|w|^{2}\right)=\left\{\left(\begin{array}{cc}
\rho & 0 \\
0 & \rho
\end{array}\right),\left(\begin{array}{cc}
0 & \eta \frac{v_{1}}{v_{2}} \\
\eta \frac{v_{2}}{v_{1}} & 0
\end{array}\right) \text { with } \rho, \eta>0\right\},
$$

and it follows that the action of $G\left(\Omega_{1}, v|w|^{2}\right)$ is not transitive on $\Omega_{1}$. This contradiction implies that exactly one of $v_{1}, v_{2}$ is non-zero, hence $D$ is linearly equivalent to the domain

$$
\left\{(z, w) \in \mathbb{C}^{2} \times \mathbb{C}: \operatorname{Im} z_{1}-|w|^{2}>0, \operatorname{Im} z_{2}>0\right\},
$$

which is biholomorphic to $B^{2} \times B^{1}$. This proves Part (2).

\section{Proof of Theorem 1.1}

By [VGP-S], [N], the manifold $M$ is biholomorphic to a Siegel domain of the second kind $S(\Omega, H)$. Since for each domain listed in Theorem 2.6 the dimension of its automorphism group is greater than $n^{2}-2$, it follows that $n \geq 4$. Also, as $M$ is not biholomorphic to $B^{n}$, we have $k \geq 2$.

Next, the following lemma rules out a large number of the remaining possibilities.

Lemma 3.1. For $n \geq 5$ one cannot have $k \geq 3$.

Proof. We will show that for $n \geq 5, k \geq 3$ the right-hand side of inequality (2.11) is strictly less than $n^{2}-2$, i.e., that for such $n, k$ the following holds:

$$
\frac{3 k^{2}}{2}-\left(2 n+\frac{5}{2}\right) k+4 n+3<0 .
$$

In order to see this, let us study the quadratic function

$$
\varphi(t):=\frac{3 t^{2}}{2}-\left(2 n+\frac{5}{2}\right) t+4 n+3
$$

on the segment $[3, n]$. Its discriminant is

$$
\mathcal{D}:=4 n^{2}-14 n-\frac{47}{4}
$$

which is easily seen to be positive for $n \geq 5$. Then the zeroes of $\varphi$ are

$$
\begin{aligned}
& t_{1}:=\frac{2 n+\frac{5}{2}-\sqrt{\mathcal{D}}}{3}, \\
& t_{2}:=\frac{2 n+\frac{5}{2}+\sqrt{\mathcal{D}}}{3} .
\end{aligned}
$$


To prove the lemma, it suffices to show that $t_{1}<3$ and $t_{2}>n$ for $n \geq 5$. Indeed, the former inequality means that

$$
2 n-\frac{13}{2}<\sqrt{\mathcal{D}}
$$

or, equivalently, that

$$
n>\frac{9}{2}
$$

which clearly holds if $n \geq 5$. Further, the inequality $t_{2}>n$ means that

or, equivalently, that

$$
n-\frac{5}{2}<\sqrt{\mathcal{D}}
$$

$$
n^{2}-3 n-6>0
$$

which is straightforward to verify for $n \geq 5$ as well.

By Lemma 3.1, in order to prove the theorem, we in fact need to consider the following three cases: (1) $k=2, n \geq 4,(2) k=3, n=4,(3) k=4, n=4$.

Case (1). Suppose that $k=2, n \geq 4$. Here $H=\left(H_{1}, H_{2}\right)$ is a pair of Hermitian forms on $\mathbb{C}^{n-2}$. After a linear change of $z$-variables, we may assume that $H_{1}$ is positive-definite. In this situation, by applying a linear change of $w$-variables, we can simultaneously diagonalize $H_{1}, H_{2}$ as

$$
H_{1}(w, w)=\|w\|^{2}, \quad H_{2}(w, w)=\sum_{j=1}^{n-2} \lambda_{j}\left|w_{j}\right|^{2} .
$$

If all the eigenvalues of $H_{2}$ are equal, $S(\Omega, H)$ is linearly equivalent either to

$$
D_{1}:=\left\{(z, w) \in \mathbb{C}^{2} \times \mathbb{C}^{n-2}: \operatorname{Im} z_{1}-\|w\|^{2}>0, \operatorname{Im} z_{2}>0\right\},
$$

or to

$$
D_{2}:=\left\{(z, w) \in \mathbb{C}^{2} \times \mathbb{C}^{n-2}: \operatorname{Im} z_{1}-\|w\|^{2}>0, \operatorname{Im} z_{2}-\|w\|^{2}>0\right\} .
$$

The domain $D_{1}$ is biholomorphic to $B^{n-1} \times B^{1}$, hence $d\left(D_{1}\right)=n^{2}+2$, which shows that $S(\Omega, H)$ cannot be equivalent to $D_{1}$. To deal with $D_{2}$, let us compute the group $G\left(\Omega_{1},\left(\|w\|^{2},\|w\|^{2}\right)\right)$. It is straightforward to see that

$$
G\left(\Omega_{1},\left(\|w\|^{2},\|w\|^{2}\right)\right)=\left\{\left(\begin{array}{cc}
\rho & 0 \\
0 & \rho
\end{array}\right),\left(\begin{array}{cc}
0 & \eta \\
\eta & 0
\end{array}\right) \text { with } \rho, \eta>0\right\}
$$

(cf. (2.16)), and it follows that the action of $G\left(\Omega_{1},\left(\|w\|^{2},\|w\|^{2}\right)\right)$ is not transitive on $\Omega_{1}$. This proves that $S(\Omega, H)$ cannot be equivalent to $D_{2}$ either. Therefore, $H_{2}$ has at least one pair of distinct eigenvalues.

Next, as $\operatorname{dim} \mathfrak{g}(\Omega)=2$, inequality (2.8) yields

$$
s \geq n^{2}-4 n \text {. }
$$

On the other hand, by (2.9), we have

$$
s \leq n^{2}-4 n+4 .
$$

More precisely, $s$ is calculated as

$$
s=n^{2}-4 n+4-2 m,
$$

where $m \geq 1$ is the number of pairs of distinct eigenvalues of $H_{2}$. Indeed, if

$$
B=\left(B_{i j}\right), \quad B_{i j}=-\overline{B_{j i}}, i, j=1, \ldots, n-2,
$$

is skew-symmetric with respect to $H_{1}$, the condition of skew-symmetricity with respect to $H_{2}$ is written as

$$
B_{i j} \lambda_{i}=-\bar{B}_{j i} \lambda_{j}, \quad i, j=1, \ldots, n-2,
$$


which leads to $B_{i j}=0$ if $\lambda_{i} \neq \lambda_{j}$.

By (3.1), (3.2) it follows that $1 \leq m \leq 2$, thus we have either $n=4$ and $\lambda_{1} \neq \lambda_{2}$ (here $m=1, s=2$ ), or $n=5$ and, upon permutation of $w$-variables, $\lambda_{1} \neq \lambda_{2}=\lambda_{3}$ (here $m=2, s=5$ ). We will now consider these two situations separately.

Case (1a). Suppose that $n=4, \lambda_{1} \neq \lambda_{2}$. Here, after a linear change of variables the domain $S(\Omega, H)$ takes the form

$$
\begin{array}{r}
D_{3}:=\left\{(z, w) \in \mathbb{C}^{2} \times \mathbb{C}^{2}: \operatorname{Im} z_{1}-\left(\alpha\left|w_{1}\right|^{2}+\beta\left|w_{2}\right|^{2}\right)>0,\right. \\
\left.\operatorname{Im} z_{2}-\left(\gamma\left|w_{1}\right|^{2}+\delta\left|w_{2}\right|^{2}\right)>0\right\},
\end{array}
$$

where $\alpha, \beta, \gamma, \delta \geq 0$ and

$$
\operatorname{det}\left(\begin{array}{ll}
\alpha & \beta \\
\gamma & \delta
\end{array}\right) \neq 0
$$

We may also assume that $\alpha>0$. If $\beta=\gamma=0$, the domain $D_{3}$ is biholomorphic to $B^{2} \times B^{2}$. Since $d\left(B^{2} \times B^{2}\right)=16=n^{2}$, we in fact have $\beta+\gamma>0$.

Lemma 3.2. If $\beta+\gamma>0$, for $\mathfrak{g}=\mathfrak{g}\left(D_{3}\right)$ one has $\mathfrak{g}_{1 / 2}=0$.

Proof. We will apply Theorem 2.4 to the cone $\Omega_{1}$ and the $\Omega_{1}$-Hermitian form

$$
\mathcal{H}\left(w, w^{\prime}\right):=\left(\alpha \bar{w}_{1} w_{1}^{\prime}+\beta \bar{w}_{2} w_{2}^{\prime}, \gamma \bar{w}_{1} w_{1}^{\prime}+\delta \bar{w}_{2} w_{2}^{\prime}\right) .
$$

Let $\Phi: \mathbb{C}^{2} \rightarrow \mathbb{C}^{2}$ be a $\mathbb{C}$-linear map:

$$
\Phi\left(z_{1}, z_{2}\right)=\left(\varphi_{1}^{1} z_{1}+\varphi_{2}^{1} z_{2}, \varphi_{1}^{2} z_{1}+\varphi_{2}^{2} z_{2}\right),
$$

where $\varphi_{j}^{i} \in \mathbb{C}$. Fixing $\mathbf{w} \in \mathbb{C}^{2}$, for $x \in \mathbb{R}^{2}$ we compute

$$
\begin{aligned}
& \mathcal{H}(\mathbf{w}, \Phi(x))=\left(\alpha \overline{\mathbf{w}}_{1}\left(\varphi_{1}^{1} x_{1}+\varphi_{2}^{1} x_{2}\right)+\beta \overline{\mathbf{w}}_{2}\left(\varphi_{1}^{2} x_{1}+\varphi_{2}^{2} x_{2}\right),\right. \\
&\left.\gamma \overline{\mathbf{w}}_{1}\left(\varphi_{1}^{1} x_{1}+\varphi_{2}^{1} x_{2}\right)+\delta \overline{\mathbf{w}}_{2}\left(\varphi_{1}^{2} x_{1}+\varphi_{2}^{2} x_{2}\right)\right)= \\
&\left(\left(\alpha \overline{\mathbf{w}}_{1} \varphi_{1}^{1}+\beta \overline{\mathbf{w}}_{2} \varphi_{1}^{2}\right) x_{1}+\left(\alpha \overline{\mathbf{w}}_{1} \varphi_{2}^{1}+\beta \overline{\mathbf{w}}_{2} \varphi_{2}^{2}\right) x_{2},\right. \\
&\left.\left(\gamma \overline{\mathbf{w}}_{1} \varphi_{1}^{1}+\delta \overline{\mathbf{w}}_{2} \varphi_{1}^{2}\right) x_{1}+\left(\gamma \overline{\mathbf{w}}_{1} \varphi_{2}^{1}+\delta \overline{\mathbf{w}}_{2} \varphi_{2}^{2}\right) x_{2}\right) .
\end{aligned}
$$

Then from formula (2.12) we see

$$
\begin{aligned}
& \Phi_{\mathbf{w}}(x)=((\left.\alpha \operatorname{Im}\left(\overline{\mathbf{w}}_{1} \varphi_{1}^{1}\right)+\beta \operatorname{Im}\left(\overline{\mathbf{w}}_{2} \varphi_{1}^{2}\right)\right) x_{1}+\left(\alpha \operatorname{Im}\left(\overline{\mathbf{w}}_{1} \varphi_{2}^{1}\right)+\beta \operatorname{Im}\left(\overline{\mathbf{w}}_{2} \varphi_{2}^{2}\right)\right) x_{2}, \\
&\left.\left(\gamma \operatorname{Im}\left(\overline{\mathbf{w}}_{1} \varphi_{1}^{1}\right)+\delta \operatorname{Im}\left(\overline{\mathbf{w}}_{2} \varphi_{1}^{2}\right)\right) x_{1}+\left(\gamma \operatorname{Im}\left(\overline{\mathbf{w}}_{1} \varphi_{2}^{1}\right)+\delta \operatorname{Im}\left(\overline{\mathbf{w}}_{2} \varphi_{2}^{2}\right)\right) x_{2}\right) .
\end{aligned}
$$

The condition that this map lies in $\mathfrak{g}\left(\Omega_{1}\right)$ for every $\mathbf{w} \in \mathbb{C}^{2}$ means

$$
\begin{aligned}
& \alpha \operatorname{Im}\left(\overline{\mathbf{w}}_{1} \varphi_{2}^{1}\right)+\beta \operatorname{Im}\left(\overline{\mathbf{w}}_{2} \varphi_{2}^{2}\right) \equiv 0, \\
& \gamma \operatorname{Im}\left(\overline{\mathbf{w}}_{1} \varphi_{1}^{1}\right)+\delta \operatorname{Im}\left(\overline{\mathbf{w}}_{2} \varphi_{1}^{2}\right) \equiv 0,
\end{aligned}
$$

which leads to the relations

$$
\varphi_{2}^{1}=0, \beta \varphi_{2}^{2}=0, \gamma \varphi_{1}^{1}=0, \delta \varphi_{1}^{2}=0
$$

(recall that $\alpha>0$ ). If each of $\beta, \gamma, \delta$ is non-zero, we see from (3.5) that $\Phi=0$, which by formula (2.13) implies $\mathfrak{g}_{1 / 2}=0$ as required.

Suppose now that $\beta=0$, hence each of $\gamma, \delta$ is non-zero. Then (3.5) yields

$$
\varphi_{1}^{1}=0, \varphi_{2}^{1}=0, \varphi_{1}^{2}=0 .
$$

Thus, $\Phi\left(z_{1}, z_{2}\right)=\left(0, \varphi_{2}^{2} z_{2}\right)$, and for $w, w^{\prime} \in \mathbb{C}^{2}$ we compute

$$
2 i \mathcal{H}\left(\Phi\left(\mathcal{H}\left(w^{\prime}, w\right)\right), w^{\prime}\right)=\left(0,2 i \gamma \delta \bar{\varphi}_{2}^{2} \bar{w}_{1} w_{1}^{\prime} w_{2}^{\prime}+2 i \delta^{2} \bar{\varphi}_{2}^{2} \bar{w}_{2}\left(w_{2}^{\prime}\right)^{2}\right) .
$$

Further, let $c$ be a symmetric $\mathbb{C}$-bilinear form on $\mathbb{C}^{2}$ with values in $\mathbb{C}^{2}$ :

$$
c(w, w)=\left(c_{11}^{1} w_{1}^{2}+2 c_{12}^{1} w_{1} w_{2}+c_{22}^{1} w_{2}^{2}, c_{11}^{2} w_{1}^{2}+2 c_{12}^{2} w_{1} w_{2}+c_{22}^{2} w_{2}^{2}\right),
$$


where $c_{i j}^{k} \in \mathbb{C}$. Then for $w, w^{\prime} \in \mathbb{C}^{2}$ we have

$$
\begin{gathered}
\mathcal{H}\left(w, c\left(w^{\prime}, w^{\prime}\right)\right)=\left(\alpha \bar{w}_{1}\left(c_{11}^{1}\left(w_{1}^{\prime}\right)^{2}+2 c_{12}^{1} w_{1}^{\prime} w_{2}^{\prime}+c_{22}^{1}\left(w_{2}^{\prime}\right)^{2}\right),\right. \\
\gamma \bar{w}_{1}\left(c_{11}^{1}\left(w_{1}^{\prime}\right)^{2}+2 c_{12}^{1} w_{1}^{\prime} w_{2}^{\prime}+c_{22}^{1}\left(w_{2}^{\prime}\right)^{2}\right)+ \\
\left.\delta \bar{w}_{2}\left(c_{11}^{2}\left(w_{1}^{\prime}\right)^{2}+2 c_{12}^{2} w_{1}^{\prime} w_{2}^{\prime}+c_{22}^{2}\left(w_{2}^{\prime}\right)^{2}\right)\right) .
\end{gathered}
$$

Comparing the right-hand sides of (3.6) and (3.7) for arbitrary $w, w^{\prime}$ as required by condition (2.13), we see that $c_{i j}^{1}=0$ for all $i, j$ hence $\varphi_{2}^{2}=0$ and therefore $\Phi=0$, $c=0$. Thus for $\beta=0$ we again have $\mathfrak{g}_{1 / 2}=0$ as claimed.

The cases $\gamma=0$ and $\delta=0$ are obtained from the case $\beta=0$ by permutation of variables.

By estimate (2.7), the second inequality in (2.5), and Lemma 3.2, we see

$$
d\left(D_{3}\right) \leq 12<14=n^{2}-2
$$

(recall that $s=2$ ). This shows that $S(\Omega, H)$ cannot in fact be equivalent to $D_{3}$, so Case (1a) contributes nothing to the classification of homogeneous Kobayashihyperbolic $n$-dimensional manifolds with automorphism group dimension $n^{2}-2$.

Remark 3.3. In Proposition A.8 in the appendix we prove that for $\beta+\gamma>0$ the component $\mathfrak{g}_{1}$ of the algebra $\mathfrak{g}=\mathfrak{g}\left(D_{3}\right)$ is also zero, which improves estimate (3.8) to $d\left(D_{3}\right) \leq 10$.

Case (1b). Suppose that $n=5$ and $\lambda_{1} \neq \lambda_{2}=\lambda_{3}$. Here, after a linear change of variables the domain $S(\Omega, H)$ takes the form

$$
\begin{array}{r}
D_{4}:=\left\{(z, w) \in \mathbb{C}^{2} \times \mathbb{C}^{3}: \operatorname{Im} z_{1}-\left(\alpha\left|w_{1}\right|^{2}+\beta\left|w_{2}\right|^{2}+\beta\left|w_{3}\right|^{2}\right)>0,\right. \\
\left.\operatorname{Im} z_{2}-\left(\gamma\left|w_{1}\right|^{2}+\delta\left|w_{2}\right|^{2}+\delta\left|w_{3}\right|^{2}\right)>0\right\},
\end{array}
$$

where $\alpha, \beta, \gamma, \delta \geq 0$ and

$$
\operatorname{det}\left(\begin{array}{cc}
\alpha & \beta \\
\gamma & \delta
\end{array}\right) \neq 0
$$

As before, we may also assume that $\alpha>0$. Then, if $\beta=\gamma=0$, the domain $D_{4}$ is biholomorphic to $B^{3} \times B^{2}$. In this case $d\left(D_{4}\right)=23=n^{2}-2$ as desired. Assume now that $\beta+\gamma>0$.

Lemma 3.4. If $\beta+\gamma>0$, for $\mathfrak{g}=\mathfrak{g}\left(D_{4}\right)$ one has $\mathfrak{g}_{1 / 2}=0$.

Proof. We will use Theorem 2.4 for the cone $\Omega_{1}$ and the $\Omega_{1}$-Hermitian form

$$
\mathcal{H}\left(w, w^{\prime}\right):=\left(\alpha \bar{w}_{1} w_{1}^{\prime}+\beta \bar{w}_{2} w_{2}^{\prime}+\beta \bar{w}_{3} w_{3}^{\prime}, \gamma \bar{w}_{1} w_{1}^{\prime}+\delta \bar{w}_{2} w_{2}^{\prime}+\delta \bar{w}_{3} w_{3}^{\prime}\right) .
$$

Let $\Phi: \mathbb{C}^{2} \rightarrow \mathbb{C}^{3}$ be a $\mathbb{C}$-linear map:

$$
\Phi\left(z_{1}, z_{2}\right)=\left(\varphi_{1}^{1} z_{1}+\varphi_{2}^{1} z_{2}, \varphi_{1}^{2} z_{1}+\varphi_{2}^{2} z_{2}, \varphi_{1}^{3} z_{1}+\varphi_{2}^{3} z_{2}\right)
$$

where $\varphi_{j}^{i} \in \mathbb{C}$. Fixing $\mathbf{w} \in \mathbb{C}^{3}$, for $x \in \mathbb{R}^{2}$ we compute

$$
\begin{gathered}
\mathcal{H}(\mathbf{w}, \Phi(x))=\left(\alpha \overline{\mathbf{w}}_{1}\left(\varphi_{1}^{1} x_{1}+\varphi_{2}^{1} x_{2}\right)+\beta \overline{\mathbf{w}}_{2}\left(\varphi_{1}^{2} x_{1}+\varphi_{2}^{2} x_{2}\right)+\beta \overline{\mathbf{w}}_{3}\left(\varphi_{1}^{3} x_{1}+\varphi_{2}^{3} x_{2}\right),\right. \\
\left.\gamma \overline{\mathbf{w}}_{1}\left(\varphi_{1}^{1} x_{1}+\varphi_{2}^{1} x_{2}\right)+\delta \overline{\mathbf{w}}_{2}\left(\varphi_{1}^{2} x_{1}+\varphi_{2}^{2} x_{2}\right)+\delta \overline{\mathbf{w}}_{3}\left(\varphi_{1}^{3} x_{1}+\varphi_{2}^{3} x_{2}\right)\right)= \\
\left(\left(\alpha \overline{\mathbf{w}}_{1} \varphi_{1}^{1}+\beta \overline{\mathbf{w}}_{2} \varphi_{1}^{2}+\beta \overline{\mathbf{w}}_{3} \varphi_{1}^{3}\right) x_{1}+\left(\alpha \overline{\mathbf{w}}_{1} \varphi_{2}^{1}+\beta \overline{\mathbf{w}}_{2} \varphi_{2}^{2}+\beta \overline{\mathbf{w}}_{3} \varphi_{2}^{3}\right) x_{2},\right. \\
\left.\left(\gamma \overline{\mathbf{w}}_{1} \varphi_{1}^{1}+\delta \overline{\mathbf{w}}_{2} \varphi_{1}^{2}+\delta \overline{\mathbf{w}}_{3} \varphi_{1}^{3}\right) x_{1}+\left(\gamma \overline{\mathbf{w}}_{1} \varphi_{2}^{1}+\delta \overline{\mathbf{w}}_{2} \varphi_{2}^{2}+\delta \overline{\mathbf{w}}_{3} \varphi_{2}^{3}\right) x_{2}\right) .
\end{gathered}
$$

Then from formula (2.12) we obtain

$$
\begin{gathered}
\Phi_{\mathbf{w}}(x)=\left(\left(\alpha \operatorname{Im}\left(\overline{\mathbf{w}}_{1} \varphi_{1}^{1}\right)+\beta \operatorname{Im}\left(\overline{\mathbf{w}}_{2} \varphi_{1}^{2}\right)+\beta \operatorname{Im}\left(\overline{\mathbf{w}}_{3} \varphi_{1}^{3}\right)\right) x_{1}+\right. \\
\left(\alpha \operatorname{Im}\left(\overline{\mathbf{w}}_{1} \varphi_{2}^{1}\right)+\beta \operatorname{Im}\left(\overline{\mathbf{w}}_{2} \varphi_{2}^{2}\right)+\beta \operatorname{Im}\left(\overline{\mathbf{w}}_{3} \varphi_{2}^{3}\right)\right) x_{2}, \\
\left(\gamma \operatorname{Im}\left(\overline{\mathbf{w}}_{1} \varphi_{1}^{1}\right)+\delta \operatorname{Im}\left(\overline{\mathbf{w}}_{2} \varphi_{1}^{2}\right)+\delta \operatorname{Im}\left(\overline{\mathbf{w}}_{3} \varphi_{1}^{3}\right)\right) x_{1}+ \\
\left.\left(\gamma \operatorname{Im}\left(\overline{\mathbf{w}}_{1} \varphi_{2}^{1}\right)+\delta \operatorname{Im}\left(\overline{\mathbf{w}}_{2} \varphi_{2}^{2}\right)+\delta \operatorname{Im}\left(\overline{\mathbf{w}}_{3} \varphi_{2}^{3}\right)\right) x_{2}\right) .
\end{gathered}
$$


The requirement that this map lies in $\mathfrak{g}\left(\Omega_{1}\right)$ for every $\mathbf{w} \in \mathbb{C}^{3}$ is equivalent to

$$
\begin{aligned}
& \alpha \operatorname{Im}\left(\overline{\mathbf{w}}_{1} \varphi_{2}^{1}\right)+\beta \operatorname{Im}\left(\overline{\mathbf{w}}_{2} \varphi_{2}^{2}\right)+\beta \operatorname{Im}\left(\overline{\mathbf{w}}_{3} \varphi_{2}^{3}\right) \equiv 0, \\
& \gamma \operatorname{Im}\left(\overline{\mathbf{w}}_{1} \varphi_{1}^{1}\right)+\delta \operatorname{Im}\left(\overline{\mathbf{w}}_{2} \varphi_{1}^{2}\right)+\delta \operatorname{Im}\left(\overline{\mathbf{w}}_{3} \varphi_{1}^{3}\right) \equiv 0,
\end{aligned}
$$

which leads to the relations

$$
\begin{aligned}
& \varphi_{2}^{1}=0, \quad \beta \varphi_{2}^{2}=0, \quad \beta \varphi_{2}^{3}=0, \\
& \gamma \varphi_{1}^{1}=0, \quad \delta \varphi_{1}^{2}=0, \quad \delta \varphi_{1}^{3}=0 .
\end{aligned}
$$

If each of $\beta, \gamma, \delta$ is non-zero, it follows from (3.11) that $\Phi=0$, which by formula (2.13) implies $\mathfrak{g}_{1 / 2}=0$ as required.

Assume now that $\beta=0$, hence each of $\gamma, \delta$ is non-zero. Then (3.11) implies

$$
\varphi_{1}^{1}=0, \varphi_{2}^{1}=0, \varphi_{1}^{2}=0, \varphi_{1}^{3}=0 .
$$

Hence, $\Phi\left(z_{1}, z_{2}\right)=\left(0, \varphi_{2}^{2} z_{2}, \varphi_{2}^{3} z_{2}\right)$, and for $w, w^{\prime} \in \mathbb{C}^{3}$ we compute

$$
\begin{aligned}
& 2 i \mathcal{H}\left(\Phi\left(\mathcal{H}\left(w^{\prime}, w\right)\right), w^{\prime}\right)=\left(0,2 i \gamma \delta \bar{\varphi}_{2}^{2} \bar{w}_{1} w_{1}^{\prime} w_{2}^{\prime}+2 i \delta^{2} \bar{\varphi}_{2}^{2} \bar{w}_{2}\left(w_{2}^{\prime}\right)^{2}+\right. \\
& \left.\quad 2 i \delta^{2} \bar{\varphi}_{2}^{2} \bar{w}_{3} w_{2}^{\prime} w_{3}^{\prime}+2 i \gamma \delta \bar{\varphi}_{2}^{3} \bar{w}_{1} w_{1}^{\prime} w_{3}^{\prime}+2 i \delta^{2} \bar{\varphi}_{2}^{3} \bar{w}_{2} w_{2}^{\prime} w_{3}^{\prime}+2 i \delta^{2} \bar{\varphi}_{2}^{3} \bar{w}_{3}\left(w_{3}^{\prime}\right)^{2}\right) .
\end{aligned}
$$

Further, let $c$ be a symmetric $\mathbb{C}$-bilinear form on $\mathbb{C}^{3}$ with values in $\mathbb{C}^{3}$ :

$$
\begin{aligned}
c(w, w)= & \left(c_{11}^{1} w_{1}^{2}+2 c_{12}^{1} w_{1} w_{2}+2 c_{13}^{1} w_{1} w_{3}+c_{22}^{1} w_{2}^{2}+2 c_{23}^{1} w_{2} w_{3}+c_{33}^{1} w_{3}^{2},\right. \\
& c_{11}^{2} w_{1}^{2}+2 c_{12}^{2} w_{1} w_{2}+2 c_{13}^{2} w_{1} w_{3}+c_{22}^{2} w_{2}^{2}+2 c_{23}^{2} w_{2} w_{3}+c_{33}^{2} w_{3}^{2}, \\
& \left.c_{11}^{3} w_{1}^{2}+2 c_{12}^{3} w_{1} w_{2}+2 c_{13}^{3} w_{1} w_{3}+c_{22}^{3} w_{2}^{2}+2 c_{23}^{3} w_{2} w_{3}+c_{33}^{3} w_{3}^{2}\right),
\end{aligned}
$$

where $c_{i j}^{k} \in \mathbb{C}$. Then for $w, w^{\prime} \in \mathbb{C}^{3}$ we have

$$
\begin{gathered}
\mathcal{H}\left(w, c\left(w^{\prime}, w^{\prime}\right)\right)=\left(\alpha \overline { w } _ { 1 } \left(c_{11}^{1}\left(w_{1}^{\prime}\right)^{2}+2 c_{12}^{1} w_{1}^{\prime} w_{2}^{\prime}+2 c_{13}^{1} w_{1}^{\prime} w_{3}^{\prime}+c_{22}^{1}\left(w_{2}^{\prime}\right)^{2}+\right.\right. \\
\left.2 c_{23}^{1} w_{2}^{\prime} w_{3}^{\prime}+c_{33}^{1}\left(w_{3}^{\prime}\right)^{2}\right), \gamma \bar{w}_{1}\left(c_{11}^{1}\left(w_{1}^{\prime}\right)^{2}+2 c_{12}^{1} w_{1}^{\prime} w_{2}^{\prime}+2 c_{13}^{1} w_{1}^{\prime} w_{3}^{\prime}+\right. \\
\left.c_{22}^{1}\left(w_{2}^{\prime}\right)^{2}+2 c_{23}^{1} w_{2}^{\prime} w_{3}^{\prime}+c_{33}^{1}\left(w_{3}^{\prime}\right)^{2}\right)+\delta \bar{w}_{2}\left(c_{11}^{2}\left(w_{1}^{\prime}\right)^{2}+2 c_{12}^{2} w_{1}^{\prime} w_{2}^{\prime}+\right. \\
\left.2 c_{13}^{2} w_{1}^{\prime} w_{3}^{\prime}+c_{22}^{2}\left(w_{2}^{\prime}\right)^{2}+2 c_{23}^{2} w_{2}^{\prime} w_{3}^{\prime}+c_{33}^{2}\left(w_{3}^{\prime}\right)^{2}\right)+\delta \bar{w}_{3}\left(c_{11}^{3}\left(w_{1}^{\prime}\right)^{2}+\right. \\
\left.\left.2 c_{12}^{3} w_{1}^{\prime} w_{2}^{\prime}+2 c_{13}^{3} w_{1}^{\prime} w_{3}^{\prime}+c_{22}^{3}\left(w_{2}^{\prime}\right)^{2}+2 c_{23}^{3} w_{2}^{\prime} w_{3}^{\prime}+c_{33}^{3}\left(w_{3}^{\prime}\right)^{2}\right)\right) .
\end{gathered}
$$

Comparing the right-hand sides of (3.12) and (3.14) for arbitrary $w, w^{\prime}$ as required by (2.13), we see that $c_{i j}^{1}=0$ for all $i, j$ hence $\varphi_{2}^{2}=0, \varphi_{2}^{3}=0$ and therefore $\Phi=0$, $c=0$. Thus for $\beta=0$ we again have $\mathfrak{g}_{1 / 2}=0$ as claimed.

Suppose next that $\gamma=0$, hence each of $\beta, \delta$ is non-zero. In this case (3.11) yields

$$
\varphi_{2}^{1}=0, \varphi_{1}^{2}=0, \varphi_{2}^{2}=0, \varphi_{1}^{3}=0, \varphi_{2}^{3}=0 .
$$

Therefore, $\Phi\left(z_{1}, z_{2}\right)=\left(\varphi_{1}^{1} z_{1}, 0,0\right)$, and for $w, w^{\prime} \in \mathbb{C}^{3}$ we find

$$
\begin{array}{r}
2 i \mathcal{H}\left(\Phi\left(\mathcal{H}\left(w^{\prime}, w\right)\right), w^{\prime}\right)=\left(2 i \alpha^{2} \bar{\varphi}_{1}^{1} \bar{w}_{1}\left(w_{1}^{\prime}\right)^{2}+2 i \alpha \beta \bar{\varphi}_{1}^{1} \bar{w}_{2} w_{1}^{\prime} w_{2}^{\prime}+\right. \\
\left.2 i \alpha \beta \bar{\varphi}_{1}^{1} \bar{w}_{3} w_{1}^{\prime} w_{3}^{\prime}, 0\right) .
\end{array}
$$

Further, for a symmetric $\mathbb{C}$-bilinear form on $\mathbb{C}^{3}$ with values in $\mathbb{C}^{3}$ as in (3.13) we see

$$
\begin{gathered}
\mathcal{H}\left(w, c\left(w^{\prime}, w^{\prime}\right)\right)=\left(\alpha \overline { w } _ { 1 } \left(c_{11}^{1}\left(w_{1}^{\prime}\right)^{2}+2 c_{12}^{1} w_{1}^{\prime} w_{2}^{\prime}+2 c_{13}^{1} w_{1}^{\prime} w_{3}^{\prime}+c_{22}^{1}\left(w_{2}^{\prime}\right)^{2}+\right.\right. \\
\left.2 c_{23}^{1} w_{2}^{\prime} w_{3}^{\prime}+c_{33}^{1}\left(w_{3}^{\prime}\right)^{2}\right)+\beta \bar{w}_{2}\left(c_{11}^{2}\left(w_{1}^{\prime}\right)^{2}+2 c_{12}^{2} w_{1}^{\prime} w_{2}^{\prime}+2 c_{13}^{2} w_{1}^{\prime} w_{3}^{\prime}+\right. \\
\left.c_{22}^{2}\left(w_{2}^{\prime}\right)^{2}+2 c_{23}^{2} w_{2}^{\prime} w_{3}^{\prime}+c_{33}^{2}\left(w_{3}^{\prime}\right)^{2}\right)+\beta \bar{w}_{3}\left(c_{11}^{3}\left(w_{1}^{\prime}\right)^{2}+2 c_{12}^{3} w_{1}^{\prime} w_{2}^{\prime}+\right. \\
\left.2 c_{13}^{3} w_{1}^{\prime} w_{3}^{\prime}+c_{22}^{3}\left(w_{2}^{\prime}\right)^{2}+2 c_{23}^{3} w_{2}^{\prime} w_{3}^{\prime}+c_{33}^{3}\left(w_{3}^{\prime}\right)^{2}\right), \delta \bar{w}_{2}\left(c_{11}^{2}\left(w_{1}^{\prime}\right)^{2}+\right. \\
\left.2 c_{12}^{2} w_{1}^{\prime} w_{2}^{\prime}+2 c_{13}^{2} w_{1}^{\prime} w_{3}^{\prime}+c_{22}^{2}\left(w_{2}^{\prime}\right)^{2}+2 c_{23}^{2} w_{2}^{\prime} w_{3}^{\prime}+c_{33}^{2}\left(w_{3}^{\prime}\right)^{2}\right)+ \\
\delta \bar{w}_{3}\left(c_{11}^{3}\left(w_{1}^{\prime}\right)^{2}+2 c_{12}^{3} w_{1}^{\prime} w_{2}^{\prime}+2 c_{13}^{3} w_{1}^{\prime} w_{3}^{\prime}+c_{22}^{3}\left(w_{2}^{\prime}\right)^{2}+\right. \\
\left.\left.2 c_{23}^{3} w_{2}^{\prime} w_{3}^{\prime}+c_{33}^{3}\left(w_{3}^{\prime}\right)^{2}\right)\right) .
\end{gathered}
$$


Comparing the right-hand sides of (3.15) and (3.16) for arbitrary $w, w^{\prime}$, we observe that $c_{i j}^{2}=0, c_{i j}^{3}=0$ for all $i, j$ hence $\varphi_{1}^{1}=0$, and therefore $\Phi=0, c=0$. Thus for $\gamma=0$ we see that $\mathfrak{g}_{1 / 2}=0$ as well.

The case $\delta=0$ is obtained from the case $\beta=0$ by permutation of variables.

By estimate (2.7), the second inequality in (2.5), and Lemma 3.4, for $\beta+\gamma>0$ we see

$$
d\left(D_{4}\right) \leq 17<23=n^{2}-2
$$

(recall that here $s=5$ ). This shows that $S(\Omega, H)$ cannot be equivalent to $D_{4}$ unless $\beta=\gamma=0$, so Case (1b) only contributes the product $B^{3} \times B^{2}$ to the classification of homogeneous Kobayashi-hyperbolic $n$-dimensional manifolds with automorphism group dimension $n^{2}-2$.

Remark 3.5. In Proposition A.9 in the appendix we prove that for $\beta+\gamma>0$ the component $\mathfrak{g}_{1}$ of the algebra $\mathfrak{g}=\mathfrak{g}\left(D_{4}\right)$ is also zero, which improves estimate $(3.17)$ to $d\left(D_{4}\right) \leq 15$.

Case (2). Suppose that $k=3, n=4$. Here $S(\Omega, H)$ is linearly equivalent either to

$$
D_{5}:=\left\{(z, w) \in \times \mathbb{C}^{3} \times \mathbb{C}: \operatorname{Im} z-v|w|^{2} \in \Omega_{2}\right\},
$$

where $v=\left(v_{1}, v_{2}, v_{3}\right)$ is a non-zero vector in $\mathbb{R}^{3}$ with non-negative entries, or to

$$
D_{6}:=\left\{(z, w) \in \times \mathbb{C}^{3} \times \mathbb{C}: \operatorname{Im} z-v|w|^{2} \in \Omega_{3}\right\},
$$

where $v=\left(v_{1}, v_{2}, v_{3}\right)$ is a vector in $\mathbb{R}^{3}$ satisfying $v_{1}^{2} \geq v_{2}^{2}+v_{3}^{2}, v_{1}>0$. We will consider these two cases separately.

Case (2a). Assume that $S(\Omega, H)$ is equivalent to the domain $D_{5}$ defined in (3.18). If only one entry of $v$ is non-zero, $D_{5}$ is biholomorphic to $B^{2} \times B^{1} \times B^{1}$. Notice that $d\left(B^{2} \times B^{1} \times B^{1}\right)=14=n^{2}-2$ as desired.

Suppose now that at least two entries of $v$ are non-zero and consider the identity component $G\left(\Omega_{2}, v|w|^{2}\right)^{\circ}$ of the group $G\left(\Omega_{2}, v|w|^{2}\right)$. As $G\left(\Omega_{2}, v|w|^{2}\right)^{\circ}$ lies in the identity component $G\left(\Omega_{2}\right)^{\circ}$ of $G\left(\Omega_{2}\right)$, every element of $G\left(\Omega_{2}, v|w|^{2}\right)^{\circ}$ is a diagonal matrix

$$
\left(\begin{array}{lll}
\lambda_{1} & 0 & 0 \\
0 & \lambda_{2} & 0 \\
0 & 0 & \lambda_{3}
\end{array}\right), \quad \lambda_{j}>0, j=1,2,3,
$$

for which $v$ is an eigenvector. Therefore, if all entries of $v$ are non-zero, then $G\left(\Omega_{2}, v|w|^{2}\right)^{\circ}$ consists of scalar matrices, and if exactly two entries of $v$, say $v_{i}$ and $v_{j}$, are non-zero, then $G\left(\Omega_{2}, v|w|^{2}\right)^{\circ}$ consists of matrices of the form (3.20) with $\lambda_{i}=\lambda_{j}$. In either situation, the action of $G\left(\Omega_{2}, v|w|^{2}\right)^{\circ}$ on $\Omega_{2}$ is not transitive. This shows that $S(\Omega, H)$ cannot be equivalent to $D_{5}$ unless exactly one entry of $v$ is non-zero, so Case (2a) only contributes the product $B^{2} \times B^{1} \times B^{1}$ to the classification of homogeneous Kobayashi-hyperbolic $n$-dimensional manifolds with automorphism group dimension $n^{2}-2$.

Case (2b). Assume now that $S(\Omega, H)$ is equivalent to the domain $D_{6}$ defined in (3.19). Suppose first that $v_{1}^{2}>v_{2}^{2}+v_{3}^{2}$, i.e., that $v \in \Omega_{3}$. As the vector $v$ is an eigenvector of every element of $G\left(\Omega_{3}, v|w|^{2}\right)$, it then follows that $G\left(\Omega_{3}, v|w|^{2}\right)$ does not act transitively on $\Omega_{3}$. This shows that in fact we have $v_{1}=\sqrt{v_{2}^{2}+v_{3}^{2}} \neq 0$, i.e., $v \in \partial \Omega_{3} \backslash\{0\}$. Further, as the group $G\left(\Omega_{3}\right)^{\circ}=\mathbb{R}_{+} \times \operatorname{SO}(1,2)^{\circ}$ acts transitively on $\partial \Omega_{3} \backslash\{0\}$, we suppose from now on that $v=(1,1,0)$.

Lemma 3.6. For $\mathfrak{g}=\mathfrak{g}\left(D_{3}\right)$ one has $\mathfrak{g}_{1 / 2}=0$. 
Proof. We will apply Theorem 2.4 to the cone $\Omega_{3}$ and the $\Omega_{3}$-Hermitian form

$$
\mathcal{H}\left(w, w^{\prime}\right):=\left(\bar{w} w^{\prime}, \bar{w} w^{\prime}, 0\right) .
$$

Let $\Phi: \mathbb{C}^{3} \rightarrow \mathbb{C}$ be a $\mathbb{C}$-linear map:

$$
\Phi\left(z_{1}, z_{2}, z_{3}\right)=\varphi_{1} z_{1}+\varphi_{2} z_{2}+\varphi_{3} z_{3}
$$

where $\varphi_{j} \in \mathbb{C}$. Fixing $\mathbf{w} \in \mathbb{C}$, for $x \in \mathbb{R}^{3}$ we compute

$$
\mathcal{H}(\mathbf{w}, \Phi(x))=\left(\overline{\mathbf{w}}\left(\varphi_{1} x_{1}+\varphi_{2} x_{2}+\varphi_{3} x_{3}\right), \overline{\mathbf{w}}\left(\varphi_{1} x_{1}+\varphi_{2} x_{2}+\varphi_{3} x_{3}\right), 0\right) .
$$

Then from formula (2.12) we see

$$
\begin{aligned}
\Phi_{\mathbf{w}}(x)=\left(\operatorname{Im}\left(\overline{\mathbf{w}} \varphi_{1}\right) x_{1}+\operatorname{Im}\left(\overline{\mathbf{w}} \varphi_{2}\right)\right. & x_{2}+\operatorname{Im}\left(\overline{\mathbf{w}} \varphi_{3}\right) x_{3} \\
& \left.\operatorname{Im}\left(\overline{\mathbf{w}} \varphi_{1}\right) x_{1}+\operatorname{Im}\left(\overline{\mathbf{w}} \varphi_{2}\right) x_{2}+\operatorname{Im}\left(\overline{\mathbf{w}} \varphi_{3}\right) x_{3}, 0\right) .
\end{aligned}
$$

Now, recall that $\mathfrak{g}\left(\Omega_{3}\right)=\mathfrak{c}\left(\mathfrak{g l}_{3}(\mathbb{R})\right) \oplus \mathfrak{o}_{1,2}$ consists of all matrices of the form

$$
\left(\begin{array}{lll}
\lambda & p & q \\
p & \lambda & r \\
q & -r & \lambda
\end{array}\right), \quad \lambda, p, q, r \in \mathbb{R} .
$$

Therefore, the condition that the map $\Phi_{\mathbf{w}}$ lies in $\mathfrak{g}\left(\Omega_{3}\right)$ for every $\mathbf{w} \in \mathbb{C}$ immediately yields

$$
\operatorname{Im}\left(\overline{\mathbf{w}} \varphi_{1}\right) \equiv 0, \operatorname{Im}\left(\overline{\mathbf{w}} \varphi_{2}\right) \equiv 0, \operatorname{Im}\left(\overline{\mathbf{w}} \varphi_{3}\right) \equiv 0,
$$

which implies $\Phi=0$. Hence, by formula (2.13) we have $\mathfrak{g}_{1 / 2}=0$ as required.

By estimate (2.7), the second inequality in (2.5), and Lemma 3.6, we see

$$
d\left(D_{6}\right) \leq 13<14=n^{2}-2
$$

(notice that here $s=1$ ). This shows that $S(\Omega, H)$ cannot be equivalent to $D_{6}$, so Case (2b) contributes nothing to the classification of homogeneous Kobayashihyperbolic $n$-dimensional manifolds with automorphism group dimension $n^{2}-2$.

Remark 3.7. In Proposition A.10 in the appendix we prove that the component $\mathfrak{g}_{1}$ of the algebra $\mathfrak{g}=\mathfrak{g}\left(D_{6}\right)$ is 1-dimensional, which improves estimate (3.23) to $d\left(D_{6}\right) \leq 11$. In fact, it is not hard to see that for the form $\mathcal{H}$ introduced in (3.21) one has $\operatorname{dim} G\left(\Omega_{3}, \mathcal{H}\right)=3$, and therefore $\operatorname{dim} \mathfrak{g}_{0}=4$. It then follows that

$$
d\left(D_{6}\right)=\operatorname{dim} \mathfrak{g}_{-1}+\operatorname{dim} \mathfrak{g}_{-1 / 2}+\operatorname{dim} \mathfrak{g}_{0}+\operatorname{dim} \mathfrak{g}_{1}=10 .
$$

Case (3). Suppose that $k=4, n=4$. In this case, after a linear change of variables $S(\Omega, H)$ turns into one of the domains

$$
\begin{aligned}
& \left\{z \in \mathbb{C}^{4}: \operatorname{Im} z \in \Omega_{4}\right\}, \\
& \left\{z \in \mathbb{C}^{4}: \operatorname{Im} z \in \Omega_{5}\right\}, \\
& \left\{z \in \mathbb{C}^{4}: \operatorname{Im} z \in \Omega_{6}\right\}
\end{aligned}
$$

and therefore is biholomorphic either to $B^{1} \times B^{1} \times B^{1} \times B^{1}$, or to $B^{1} \times T_{3}$, or to $T_{4}$, where $T_{3}$ and $T_{4}$ are the tube domains defined in (1.1), (1.2). The dimensions of the automorphism groups of these domains are 12, 13, 15, respectively. As none of the numbers is equal to $14=n^{2}-2$, Case (3) contributes nothing to the classification of homogeneous Kobayashi-hyperbolic $n$-dimensional manifolds with automorphism group dimension $n^{2}-2$.

The proof of Theorem 1.1 is now complete. 


\section{APPENDIX}

Here we show that for the domains $D_{3}, D_{4}$ introduced in (3.3), (3.9), respectively, we have $\mathfrak{g}_{1}=0$ if $\beta+\gamma>0$. In addition, we prove that for the domain $D_{6}$ defined in (3.19) one has $\operatorname{dim} \mathfrak{g}_{1}=1$ if $v \in \partial \Omega_{3} \backslash\{0\}$. These facts can be utilized for extending our classifications in Theorems 1.1, 1.2 to automorphism group dimensions less than the critical dimension $n^{2}-2$. The proofs below are also independently interesting as they contain explicit computations with the fairly bulky formulas supplied by Theorem 2.5, which is rarely seen in the literature.

We start with the domain $D_{3}$.

Proposition A.8. If $\beta+\gamma>0$, for $\mathfrak{g}=\mathfrak{g}\left(D_{3}\right)$ one has $\mathfrak{g}_{1}=0$.

Proof. We will utilize Theorem 2.5 for the cone $\Omega_{1}$ and the $\Omega_{1}$-Hermitian form $\mathcal{H}$ given by (3.4). Consider a symmetric $\mathbb{R}$-bilinear form on $\mathbb{R}^{2}$ with values in $\mathbb{R}^{2}$ :

$$
a(x, x)=\left(a_{11}^{1} x_{1}^{2}+2 a_{12}^{1} x_{1} x_{2}+a_{22}^{1} x_{2}^{2}, a_{11}^{2} x_{1}^{2}+2 a_{12}^{2} x_{1} x_{2}+a_{22}^{2} x_{2}^{2}\right),
$$

where $a_{i j}^{k} \in \mathbb{R}$. Then for a fixed $\mathbf{x} \in \mathbb{R}^{2}$ from (2.14) we compute

$$
\begin{aligned}
& A_{\mathbf{x}}(x)=\left(a_{11}^{1} \mathbf{x}_{1} x_{1}+a_{12}^{1} \mathbf{x}_{1} x_{2}+a_{12}^{1} \mathbf{x}_{2} x_{1}+a_{22}^{1} \mathbf{x}_{2} x_{2},\right. \\
& \left.a_{11}^{2} \mathbf{x}_{1} x_{1}+a_{12}^{2} \mathbf{x}_{1} x_{2}+a_{12}^{2} \mathbf{x}_{2} x_{1}+a_{22}^{2} \mathbf{x}_{2} x_{2}\right)= \\
& \left(\left(a_{11}^{1} \mathbf{x}_{1}+a_{12}^{1} \mathbf{x}_{2}\right) x_{1}+\left(a_{12}^{1} \mathbf{x}_{1}+a_{22}^{1} \mathbf{x}_{2}\right) x_{2},\left(a_{11}^{2} \mathbf{x}_{1}+a_{12}^{2} \mathbf{x}_{2}\right) x_{1}+\left(a_{12}^{2} \mathbf{x}_{1}+a_{22}^{2} \mathbf{x}_{2}\right) x_{2}\right),
\end{aligned}
$$

where $x \in \mathbb{R}^{2}$. The condition that this map lies in $\mathfrak{g}\left(\Omega_{1}\right)$ for every $\mathbf{x} \in \mathbb{R}^{2}$ is equivalent to

$$
\begin{aligned}
& a_{12}^{1} \mathbf{x}_{1}+a_{22}^{1} \mathbf{x}_{2} \equiv 0 \\
& a_{11}^{2} \mathbf{x}_{1}+a_{12}^{2} \mathbf{x}_{2} \equiv 0
\end{aligned}
$$

which implies

$$
a_{12}^{1}=0, \quad a_{22}^{1}=0, \quad a_{11}^{2}=0, \quad a_{12}^{2}=0
$$

Therefore,

$$
A_{\mathbf{x}}(x)=\left(a_{11}^{1} \mathbf{x}_{1} x_{1}, a_{22}^{2} \mathbf{x}_{2} x_{2}\right)
$$

Next, let $b: \mathbb{C}^{2} \times \mathbb{C}^{2} \rightarrow \mathbb{C}^{2}$ be a $\mathbb{C}$-bilinear map:

$$
\begin{aligned}
b(z, w)=\left(b_{11}^{1} z_{1} w_{1}+b_{12}^{1} z_{1} w_{2}+\right. & b_{21}^{1} z_{2} w_{1}+b_{22}^{1} z_{2} w_{2} \\
& \left.b_{11}^{2} z_{1} w_{1}+b_{12}^{2} z_{1} w_{2}+b_{21}^{2} z_{2} w_{1}+b_{22}^{2} z_{2} w_{2}\right),
\end{aligned}
$$

where $b_{i j}^{k} \in \mathbb{C}$. Then for a fixed $\mathbf{x} \in \mathbb{R}^{2}$ from (2.15) we find

$$
\begin{aligned}
B_{\mathbf{x}}(w)=\frac{1}{2}\left(\left(b_{11}^{1} \mathbf{x}_{1}+b_{21}^{1} \mathbf{x}_{2}\right) w_{1}+\right. & \left(b_{12}^{1} \mathbf{x}_{1}+b_{22}^{1} \mathbf{x}_{2}\right) w_{2} \\
& \left.\left(b_{11}^{2} \mathbf{x}_{1}+b_{21}^{2} \mathbf{x}_{2}\right) w_{1}+\left(b_{12}^{2} \mathbf{x}_{1}+b_{22}^{2} \mathbf{x}_{2}\right) w_{2}\right) .
\end{aligned}
$$

The condition that $\operatorname{Im} \operatorname{tr} B_{\mathbf{x}}=0$ for all $\mathbf{x} \in \mathbb{R}^{2}$ in (i) in Theorem 2.5 means

$$
\operatorname{Im}\left(\left(b_{11}^{1}+b_{12}^{2}\right) \mathbf{x}_{1}+\left(b_{21}^{1}+b_{22}^{2}\right) \mathbf{x}_{2}\right) \equiv 0,
$$

which leads, in particular, to

$$
\operatorname{Im}\left(b_{21}^{1}+b_{22}^{2}\right)=0 .
$$


Further, for every fixed pair $\mathbf{w}, \mathbf{w}^{\prime} \in \mathbb{C}^{2}$ we compute

$$
\begin{gathered}
\mathcal{H}\left(\mathbf{w}^{\prime}, b(x, \mathbf{w})\right)=\left(\alpha \overline{\mathbf{w}}_{1}^{\prime}\left(b_{11}^{1} \mathbf{w}_{1} x_{1}+b_{12}^{1} \mathbf{w}_{2} x_{1}+b_{21}^{1} \mathbf{w}_{1} x_{2}+b_{22}^{1} \mathbf{w}_{2} x_{2}\right)+\right. \\
\beta \overline{\mathbf{w}}_{2}^{\prime}\left(b_{11}^{2} \mathbf{w}_{1} x_{1}+b_{12}^{2} \mathbf{w}_{2} x_{1}+b_{21}^{2} \mathbf{w}_{1} x_{2}+b_{22}^{2} \mathbf{w}_{2} x_{2}\right) \\
\gamma \overline{\mathbf{w}}_{1}^{\prime}\left(b_{11}^{1} \mathbf{w}_{1} x_{1}+b_{12}^{1} \mathbf{w}_{2} x_{1}+b_{21}^{1} \mathbf{w}_{1} x_{2}+b_{22}^{1} \mathbf{w}_{2} x_{2}\right)+ \\
\left.\delta \overline{\mathbf{w}}_{2}^{\prime}\left(b_{11}^{2} \mathbf{w}_{1} x_{1}+b_{12}^{2} \mathbf{w}_{2} x_{1}+b_{21}^{2} \mathbf{w}_{1} x_{2}+b_{22}^{2} \mathbf{w}_{2} x_{2}\right)\right)= \\
\left(\left(\alpha \overline{\mathbf{w}}_{1}^{\prime}\left(b_{11}^{1} \mathbf{w}_{1}+b_{12}^{1} \mathbf{w}_{2}\right)+\beta \overline{\mathbf{w}}_{2}^{\prime}\left(b_{11}^{2} \mathbf{w}_{1}+b_{12}^{2} \mathbf{w}_{2}\right)\right) x_{1}+\right. \\
\left(\alpha \overline{\mathbf{w}}_{1}^{\prime}\left(b_{21}^{1} \mathbf{w}_{1}+b_{22}^{1} \mathbf{w}_{2}\right)+\beta \overline{\mathbf{w}}_{2}^{\prime}\left(b_{21}^{2} \mathbf{w}_{1}+b_{22}^{2} \mathbf{w}_{2}\right)\right) x_{2} \\
\left(\gamma \overline{\mathbf{w}}_{1}^{\prime}\left(b_{11}^{1} \mathbf{w}_{1}+b_{12}^{1} \mathbf{w}_{2}\right)+\delta \overline{\mathbf{w}}_{2}^{\prime}\left(b_{11}^{2} \mathbf{w}_{1}+b_{12}^{2} \mathbf{w}_{2}\right)\right) x_{1}+ \\
\left.\left(\gamma \overline{\mathbf{w}}_{1}^{\prime}\left(b_{21}^{1} \mathbf{w}_{1}+b_{22}^{1} \mathbf{w}_{2}\right)+\delta \overline{\mathbf{w}}_{2}^{\prime}\left(b_{21}^{2} \mathbf{w}_{1}+b_{22}^{2} \mathbf{w}_{2}\right)\right) x_{2}\right) .
\end{gathered}
$$

Then from (ii) of Theorem 2.5 we obtain

$$
\begin{gathered}
B_{\mathbf{w}, \mathbf{w}^{\prime}}(x)=\left(\operatorname{Im}\left(\alpha \overline{\mathbf{w}}_{1}^{\prime}\left(b_{11}^{1} \mathbf{w}_{1}+b_{12}^{1} \mathbf{w}_{2}\right)+\beta \overline{\mathbf{w}}_{2}^{\prime}\left(b_{11}^{2} \mathbf{w}_{1}+b_{12}^{2} \mathbf{w}_{2}\right)\right) x_{1}+\right. \\
\operatorname{Im}\left(\alpha \overline{\mathbf{w}}_{1}^{\prime}\left(b_{21}^{1} \mathbf{w}_{1}+b_{22}^{1} \mathbf{w}_{2}\right)+\beta \overline{\mathbf{w}}_{2}^{\prime}\left(b_{21}^{2} \mathbf{w}_{1}+b_{22}^{2} \mathbf{w}_{2}\right)\right) x_{2}, \\
\operatorname{Im}\left(\gamma \overline{\mathbf{w}}_{1}^{\prime}\left(b_{11}^{1} \mathbf{w}_{1}+b_{12}^{1} \mathbf{w}_{2}\right)+\delta \overline{\mathbf{w}}_{2}^{\prime}\left(b_{11}^{2} \mathbf{w}_{1}+b_{12}^{2} \mathbf{w}_{2}\right)\right) x_{1}+ \\
\left.\operatorname{Im}\left(\gamma \overline{\mathbf{w}}_{1}^{\prime}\left(b_{21}^{1} \mathbf{w}_{1}+b_{22}^{1} \mathbf{w}_{2}\right)+\delta \overline{\mathbf{w}}_{2}^{\prime}\left(b_{21}^{2} \mathbf{w}_{1}+b_{22}^{2} \mathbf{w}_{2}\right)\right) x_{2}\right) .
\end{gathered}
$$

The condition that this map lies in $\mathfrak{g}\left(\Omega_{1}\right)$ for all $\mathbf{w}, \mathbf{w}^{\prime} \in \mathbb{C}^{2}$ means

$$
\begin{aligned}
& \operatorname{Im}\left(\alpha \overline{\mathbf{w}}_{1}^{\prime}\left(b_{21}^{1} \mathbf{w}_{1}+b_{22}^{1} \mathbf{w}_{2}\right)+\beta \overline{\mathbf{w}}_{2}^{\prime}\left(b_{21}^{2} \mathbf{w}_{1}+b_{22}^{2} \mathbf{w}_{2}\right)\right) \equiv 0, \\
& \operatorname{Im}\left(\gamma \overline{\mathbf{w}}_{1}^{\prime}\left(b_{11}^{1} \mathbf{w}_{1}+b_{12}^{1} \mathbf{w}_{2}\right)+\delta \overline{\mathbf{w}}_{2}^{\prime}\left(b_{11}^{2} \mathbf{w}_{1}+b_{12}^{2} \mathbf{w}_{2}\right)\right) \equiv 0,
\end{aligned}
$$

which yields

$$
\begin{array}{llll}
b_{21}^{1}=0, & b_{22}^{1}=0, & \beta b_{21}^{2}=0, & \beta b_{22}^{2}=0, \\
\gamma b_{11}^{1}=0, & \gamma b_{12}^{1}=0, & \delta b_{11}^{2}=0, & \delta b_{12}^{2}=0 .
\end{array}
$$

If each of $\beta, \gamma, \delta$ is non-zero, it follows from (A.5) that $b=0$, therefore $B_{\mathbf{x}}=0$ for all $\mathbf{x} \in \mathbb{R}^{2}$, and the requirement that $B_{\mathbf{x}}$ is associated to $A_{\mathbf{x}}$ with respect to $\mathcal{H}$ for every $\mathbf{x} \in \mathbb{R}^{2}$ (see condition (i) in Theorem 2.5) implies $a=0$. Thus, $\mathfrak{g}_{1}=0$ as claimed.

Assume now that $\beta=0$, hence each of $\gamma, \delta$ is non-zero, so by (A.5) we have

$$
b_{11}^{1}=0, b_{12}^{1}=0, b_{21}^{1}=0, b_{22}^{1}=0, b_{11}^{2}=0, b_{12}^{2}=0,
$$

and (A.4) yields

$$
\operatorname{Im} b_{22}^{2}=0 \text {. }
$$

Thus,

$$
B_{\mathbf{x}}(w)=\frac{1}{2}\left(0, b_{21}^{2} \mathbf{x}_{2} w_{1}+b_{22}^{2} \mathbf{x}_{2} w_{2}\right) .
$$

We will now use the requirement that $B_{\mathbf{x}}$ is associated to $A_{\mathbf{x}}$ with respect to $\mathcal{H}$ for every $\mathbf{x} \in \mathbb{R}^{2}$ as in (i) in Theorem 2.5. On the one hand, from (A.3) we have

$$
A_{\mathbf{x}} \mathcal{H}\left(w, w^{\prime}\right)=\left(a_{11}^{1} \mathbf{x}_{1} \alpha \bar{w}_{1} w_{1}^{\prime}, a_{22}^{2} \mathbf{x}_{2}\left(\gamma \bar{w}_{1} w_{1}^{\prime}+\delta \bar{w}_{2} w_{2}^{\prime}\right)\right) .
$$

On the other hand, from (A.7) one obtains

$$
\begin{array}{r}
\mathcal{H}\left(B_{\mathbf{x}}(w), w^{\prime}\right)+\mathcal{H}\left(w, B_{\mathbf{x}}\left(w^{\prime}\right)\right)=\frac{1}{2}\left(0, \delta\left(\bar{b}_{21}^{2} \mathbf{x}_{2} \bar{w}_{1}+\bar{b}_{22}^{2} \mathbf{x}_{2} \bar{w}_{2}\right) w_{2}^{\prime}+\right. \\
\left.\delta \bar{w}_{2}\left(b_{21}^{2} \mathbf{x}_{2} w_{1}^{\prime}+b_{22}^{2} \mathbf{x}_{2} w_{2}^{\prime}\right)\right) .
\end{array}
$$

Comparing (A.8) and (A.9), we deduce

$$
a_{11}^{1}=0, a_{22}^{2}=0, b_{21}^{2}=0, \operatorname{Re} b_{22}^{2}=0 .
$$

By (A.6) and (A.10) we see that $a=0, b=0$, which shows that $\mathfrak{g}_{1}=0$ as claimed. 
The cases $\gamma=0$ and $\delta=0$ are obtained from the case $\beta=0$ by permutation of variables.

Next, we will deduce an analogous fact for the domain $D_{4}$.

Proposition A.9. If $\beta+\gamma>0$, for $\mathfrak{g}=\mathfrak{g}\left(D_{4}\right)$ one has $\mathfrak{g}_{1}=0$.

Proof. We will apply Theorem 2.5 to the cone $\Omega_{1}$ and $\Omega_{1}$-Hermitian form (3.10). Consider a symmetric $\mathbb{R}$-bilinear form on $\mathbb{R}^{2}$ with values in $\mathbb{R}^{2}$ (see (A.1)). The proof of Proposition A.8 shows that the coefficients of this form satisfy (A.2). Hence, for every fixed $\mathbf{x} \in \mathbb{R}^{2}$ the map $A_{\mathbf{x}}$ defined in (2.14) is given by formula (A.3).

Next, let $b: \mathbb{C}^{2} \times \mathbb{C}^{3} \rightarrow \mathbb{C}^{3}$ be a $\mathbb{C}$-bilinear map:

$$
\begin{aligned}
b(z, w)= & \left(b_{11}^{1} z_{1} w_{1}+b_{12}^{1} z_{1} w_{2}+b_{13}^{1} z_{1} w_{3}+b_{21}^{1} z_{2} w_{1}+b_{22}^{1} z_{2} w_{2}+b_{23}^{1} z_{2} w_{3},\right. \\
& b_{11}^{2} z_{1} w_{1}+b_{12}^{2} z_{1} w_{2}+b_{13}^{2} z_{1} w_{3}+b_{21}^{2} z_{2} w_{1}+b_{22}^{2} z_{2} w_{2}+b_{23}^{2} z_{2} w_{3}, \\
& \left.b_{11}^{3} z_{1} w_{1}+b_{12}^{3} z_{1} w_{2}+b_{13}^{3} z_{1} w_{3}+b_{21}^{3} z_{2} w_{1}+b_{22}^{3} z_{2} w_{2}+b_{23}^{3} z_{2} w_{3}\right)
\end{aligned}
$$

where $b_{i j}^{k} \in \mathbb{C}$. Then for a fixed $\mathbf{x} \in \mathbb{R}^{2}$ from (2.15) we compute

$$
\begin{array}{r}
B_{\mathbf{x}}(w)=\frac{1}{2}\left(\left(b_{11}^{1} \mathbf{x}_{1}+b_{21}^{1} \mathbf{x}_{2}\right) w_{1}+\left(b_{12}^{1} \mathbf{x}_{1}+b_{22}^{1} \mathbf{x}_{2}\right) w_{2}+\left(b_{13}^{1} \mathbf{x}_{1}+b_{23}^{1} \mathbf{x}_{2}\right) w_{3}\right. \\
\left(b_{11}^{2} \mathbf{x}_{1}+b_{21}^{2} \mathbf{x}_{2}\right) w_{1}+\left(b_{12}^{2} \mathbf{x}_{1}+b_{22}^{2} \mathbf{x}_{2}\right) w_{2}+\left(b_{13}^{2} \mathbf{x}_{1}+b_{23}^{2} \mathbf{x}_{2}\right) w_{3} \\
\left.\left(b_{11}^{3} \mathbf{x}_{1}+b_{21}^{3} \mathbf{x}_{2}\right) w_{1}+\left(b_{12}^{3} \mathbf{x}_{1}+b_{22}^{3} \mathbf{x}_{2}\right) w_{2}+\left(b_{13}^{3} \mathbf{x}_{1}+b_{23}^{3} \mathbf{x}_{2}\right) w_{3}\right) .
\end{array}
$$

The condition that $\operatorname{Im} \operatorname{tr} B_{\mathbf{x}}=0$ for all $\mathbf{x} \in \mathbb{R}^{2}$ in (i) in Theorem 2.5 means

$$
\operatorname{Im}\left(\left(b_{11}^{1}+b_{12}^{2}+b_{13}^{3}\right) \mathbf{x}_{1}+\left(b_{21}^{1}+b_{22}^{2}+b_{23}^{3}\right) \mathbf{x}_{2}\right) \equiv 0
$$

which leads, in particular, to

$$
\operatorname{Im}\left(b_{11}^{1}+b_{12}^{2}+b_{13}^{3}\right)=0 .
$$

Further, for every fixed pair $\mathbf{w}, \mathbf{w}^{\prime} \in \mathbb{C}^{3}$ we find

$$
\begin{aligned}
& \mathcal{H}\left(\mathbf{w}^{\prime}, b(x, \mathbf{w})\right)=\left(\alpha \overline { \mathbf { w } } _ { 1 } ^ { \prime } \left(b_{11}^{1} \mathbf{w}_{1} x_{1}+b_{12}^{1} \mathbf{w}_{2} x_{1}+b_{13}^{1} \mathbf{w}_{3} x_{1}+b_{21}^{1} \mathbf{w}_{1} x_{2}+b_{22}^{1} \mathbf{w}_{2} x_{2}+\right.\right. \\
& \left.b_{23}^{1} \mathbf{w}_{3} x_{2}\right)+\beta \overline{\mathbf{w}}_{2}^{\prime}\left(b_{11}^{2} \mathbf{w}_{1} x_{1}+b_{12}^{2} \mathbf{w}_{2} x_{1}+b_{13}^{2} \mathbf{w}_{3} x_{1}+b_{21}^{2} \mathbf{w}_{1} x_{2}+b_{22}^{2} \mathbf{w}_{2} x_{2}+\right. \\
& \left.b_{23}^{2} \mathbf{w}_{3} x_{2}\right)+\beta \overline{\mathbf{w}}_{3}^{\prime}\left(b_{11}^{3} \mathbf{w}_{1} x_{1}+b_{12}^{3} \mathbf{w}_{2} x_{1}+b_{13}^{3} \mathbf{w}_{3} x_{1}+b_{21}^{3} \mathbf{w}_{1} x_{2}+b_{22}^{3} \mathbf{w}_{2} x_{2}+\right. \\
& \left.b_{23}^{3} \mathbf{w}_{3} x_{2}\right), \gamma \overline{\mathbf{w}}_{1}^{\prime}\left(b_{11}^{1} \mathbf{w}_{1} x_{1}+b_{12}^{1} \mathbf{w}_{2} x_{1}+b_{13}^{1} \mathbf{w}_{3} x_{1}+b_{21}^{1} \mathbf{w}_{1} x_{2}+b_{22}^{1} \mathbf{w}_{2} x_{2}+\right. \\
& \left.b_{23}^{1} \mathbf{w}_{3} x_{2}\right)+\delta \overline{\mathbf{w}}_{2}^{\prime}\left(b_{11}^{2} \mathbf{w}_{1} x_{1}+b_{12}^{2} \mathbf{w}_{2} x_{1}+b_{13}^{2} \mathbf{w}_{3} x_{1}+b_{21}^{2} \mathbf{w}_{1} x_{2}+b_{22}^{2} \mathbf{w}_{2} x_{2}+\right. \\
& \left.b_{23}^{2} \mathbf{w}_{3} x_{2}\right)+\delta \overline{\mathbf{w}}_{3}^{\prime}\left(b_{11}^{3} \mathbf{w}_{1} x_{1}+b_{12}^{3} \mathbf{w}_{2} x_{1}+b_{13}^{3} \mathbf{w}_{3} x_{1}+b_{21}^{3} \mathbf{w}_{1} x_{2}+b_{22}^{3} \mathbf{w}_{2} x_{2}+\right. \\
& \left.\left.b_{23}^{3} \mathbf{w}_{3} x_{2}\right)\right)=\left(\left(\alpha \overline{\mathbf{w}}_{1}^{\prime}\left(b_{11}^{1} \mathbf{w}_{1}+b_{12}^{1} \mathbf{w}_{2}+b_{13}^{1} \mathbf{w}_{3}\right)+\beta \overline{\mathbf{w}}_{2}^{\prime}\left(b_{11}^{2} \mathbf{w}_{1}+b_{12}^{2} \mathbf{w}_{2}+\right.\right.\right. \\
& \left.\left.b_{13}^{2} \mathbf{w}_{3}\right)+\beta \overline{\mathbf{w}}_{3}^{\prime}\left(b_{11}^{3} \mathbf{w}_{1}+b_{12}^{3} \mathbf{w}_{2}+b_{13}^{3} \mathbf{w}_{3}\right)\right) x_{1}+\left(\alpha \overline { \mathbf { w } } _ { 1 } ^ { \prime } \left(b_{21}^{1} \mathbf{w}_{1}+b_{22}^{1} \mathbf{w}_{2}+\right.\right. \\
& \left.b_{23}^{1} \mathbf{w}_{3}\right)+\beta \overline{\mathbf{w}}_{2}^{\prime}\left(b_{21}^{2} \mathbf{w}_{1}+b_{22}^{2} \mathbf{w}_{2}+b_{23}^{2} \mathbf{w}_{3}\right)+\beta \overline{\mathbf{w}}_{3}^{\prime}\left(b_{21}^{3} \mathbf{w}_{1}+b_{22}^{3} \mathbf{w}_{2}+\right. \\
& \left.\left.b_{23}^{3} \mathbf{w}_{3}\right)\right) x_{2},\left(\gamma \overline{\mathbf{w}}_{1}^{\prime}\left(b_{11}^{1} \mathbf{w}_{1}+b_{12}^{1} \mathbf{w}_{2}+b_{13}^{1} \mathbf{w}_{3}\right)+\delta \overline{\mathbf{w}}_{2}^{\prime}\left(b_{11}^{2} \mathbf{w}_{1}+b_{12}^{2} \mathbf{w}_{2}+\right.\right. \\
& \left.\left.b_{13}^{2} \mathbf{w}_{3}\right)+\delta \overline{\mathbf{w}}_{3}^{\prime}\left(b_{11}^{3} \mathbf{w}_{1}+b_{12}^{3} \mathbf{w}_{2}+b_{13}^{3} \mathbf{w}_{3}\right)\right) x_{1}+\left(\gamma \overline { \mathbf { w } } _ { 1 } ^ { \prime } \left(b_{21}^{1} \mathbf{w}_{1}+b_{22}^{1} \mathbf{w}_{2}+\right.\right. \\
& \left.b_{23}^{1} \mathbf{w}_{3}\right)+\delta \overline{\mathbf{w}}_{2}^{\prime}\left(b_{21}^{2} \mathbf{w}_{1}+b_{22}^{2} \mathbf{w}_{2}+b_{23}^{2} \mathbf{w}_{3}\right)+\delta \overline{\mathbf{w}}_{3}^{\prime}\left(b_{21}^{3} \mathbf{w}_{1}+b_{22}^{3} \mathbf{w}_{2}+\right. \\
& \left.\left.\left.b_{23}^{3} \mathbf{w}_{3}\right)\right) x_{2}\right) \text {. }
\end{aligned}
$$


Then from (ii) of Theorem 2.5 we see

$$
\begin{gathered}
B_{\mathbf{w}, \mathbf{w}^{\prime}}(x)=\left(\operatorname { I m } \left(\alpha \overline{\mathbf{w}}_{1}^{\prime}\left(b_{11}^{1} \mathbf{w}_{1}+b_{12}^{1} \mathbf{w}_{2}+b_{13}^{1} \mathbf{w}_{3}\right)+\beta \overline{\mathbf{w}}_{2}^{\prime}\left(b_{11}^{2} \mathbf{w}_{1}+b_{12}^{2} \mathbf{w}_{2}+b_{13}^{2} \mathbf{w}_{3}\right)+\right.\right. \\
\left.\beta \overline{\mathbf{w}}_{3}^{\prime}\left(b_{11}^{3} \mathbf{w}_{1}+b_{12}^{3} \mathbf{w}_{2}+b_{13}^{3} \mathbf{w}_{3}\right)\right) x_{1}+\operatorname{Im}\left(\alpha \overline{\mathbf{w}}_{1}^{\prime}\left(b_{21}^{1} \mathbf{w}_{1}+b_{22}^{1} \mathbf{w}_{2}+b_{23}^{1} \mathbf{w}_{3}\right)+\right. \\
\left.\beta \overline{\mathbf{w}}_{2}^{\prime}\left(b_{21}^{2} \mathbf{w}_{1}+b_{22}^{2} \mathbf{w}_{2}+b_{23}^{2} \mathbf{w}_{3}\right)+\beta \overline{\mathbf{w}}_{3}^{\prime}\left(b_{21}^{3} \mathbf{w}_{1}+b_{22}^{3} \mathbf{w}_{2}+b_{23}^{3} \mathbf{w}_{3}\right)\right) x_{2} \\
\operatorname{Im}\left(\gamma \overline{\mathbf{w}}_{1}^{\prime}\left(b_{11}^{1} \mathbf{w}_{1}+b_{12}^{1} \mathbf{w}_{2}+b_{13}^{1} \mathbf{w}_{3}\right)+\delta \overline{\mathbf{w}}_{2}^{\prime}\left(b_{11}^{2} \mathbf{w}_{1}+b_{12}^{2} \mathbf{w}_{2}+b_{13}^{2} \mathbf{w}_{3}\right)+\right. \\
\left.\delta \overline{\mathbf{w}}_{3}^{\prime}\left(b_{11}^{3} \mathbf{w}_{1}+b_{12}^{3} \mathbf{w}_{2}+b_{13}^{3} \mathbf{w}_{3}\right)\right) x_{1}+\operatorname{Im}\left(\gamma \overline{\mathbf{w}}_{1}^{\prime}\left(b_{21}^{1} \mathbf{w}_{1}+b_{22}^{1} \mathbf{w}_{2}+b_{23}^{1} \mathbf{w}_{3}\right)+\right. \\
\left.\left.\delta \overline{\mathbf{w}}_{2}^{\prime}\left(b_{21}^{2} \mathbf{w}_{1}+b_{22}^{2} \mathbf{w}_{2}+b_{23}^{2} \mathbf{w}_{3}\right)+\delta \overline{\mathbf{w}}_{3}^{\prime}\left(b_{21}^{3} \mathbf{w}_{1}+b_{22}^{3} \mathbf{w}_{2}+b_{23}^{3} \mathbf{w}_{3}\right)\right) x_{2}\right) .
\end{gathered}
$$

The condition that this map lies in $\mathfrak{g}\left(\Omega_{1}\right)$ for all $\mathbf{w}, \mathbf{w}^{\prime} \in \mathbb{C}^{3}$ is equivalent to

$$
\begin{gathered}
\operatorname{Im}\left(\alpha \overline{\mathbf{w}}_{1}^{\prime}\left(b_{21}^{1} \mathbf{w}_{1}+b_{22}^{1} \mathbf{w}_{2}+b_{23}^{1} \mathbf{w}_{3}\right)+\beta \overline{\mathbf{w}}_{2}^{\prime}\left(b_{21}^{2} \mathbf{w}_{1}+b_{22}^{2} \mathbf{w}_{2}+b_{23}^{2} \mathbf{w}_{3}\right)+\right. \\
\left.\beta \overline{\mathbf{w}}_{3}^{\prime}\left(b_{21}^{3} \mathbf{w}_{1}+b_{22}^{3} \mathbf{w}_{2}+b_{23}^{3} \mathbf{w}_{3}\right)\right) \equiv 0, \\
\operatorname{Im}\left(\gamma \overline{\mathbf{w}}_{1}^{\prime}\left(b_{11}^{1} \mathbf{w}_{1}+b_{12}^{1} \mathbf{w}_{2}+b_{13}^{1} \mathbf{w}_{3}\right)+\delta \overline{\mathbf{w}}_{2}^{\prime}\left(b_{11}^{2} \mathbf{w}_{1}+b_{12}^{2} \mathbf{w}_{2}+b_{13}^{2} \mathbf{w}_{3}\right)+\right. \\
\left.\delta \overline{\mathbf{w}}_{3}^{\prime}\left(b_{11}^{3} \mathbf{w}_{1}+b_{12}^{3} \mathbf{w}_{2}+b_{13}^{3} \mathbf{w}_{3}\right)\right) \equiv 0,
\end{gathered}
$$

which yields

$$
\begin{aligned}
& b_{21}^{1}=0, \quad b_{22}^{1}=0, \quad b_{23}^{1}=0, \quad \beta b_{21}^{2}=0, \quad \beta b_{22}^{2}=0, \quad \beta b_{23}^{2}=0, \\
& \beta b_{21}^{3}=0, \quad \beta b_{22}^{3}=0, \quad \beta b_{23}^{3}=0, \quad \gamma b_{11}^{1}=0, \quad \gamma b_{12}^{1}=0, \quad \gamma b_{13}^{1}=0 \text {, } \\
& \delta b_{11}^{2}=0, \quad \delta b_{12}^{2}=0, \quad \delta b_{13}^{2}=0, \quad \delta b_{11}^{3}=0, \quad \delta b_{12}^{3}=0, \quad \delta b_{13}^{3}=0 .
\end{aligned}
$$

If each of $\beta, \gamma, \delta$ is non-zero, it follows from (A.12) that $b=0$, therefore $B_{\mathbf{x}}=0$ for all $\mathbf{x} \in \mathbb{R}^{2}$, and the requirement that $B_{\mathbf{x}}$ is associated to $A_{\mathbf{x}}$ with respect to $\mathcal{H}$ for every $\mathbf{x} \in \mathbb{R}^{2}$ (see condition (i) in Theorem 2.5) implies $a=0$. Thus, $\mathfrak{g}_{1}=0$ as required.

Assume now that $\beta=0$, hence each of $\gamma, \delta$ is non-zero, and by (A.12) we have

$$
\begin{aligned}
& b_{11}^{1}=0, b_{12}^{1}=0, b_{13}^{1}=0, b_{21}^{1}=0, b_{22}^{1}=0, b_{23}^{1}=0, \\
& b_{11}^{2}=0, b_{12}^{2}=0, b_{13}^{2}=0, b_{11}^{3}=0, b_{12}^{3}=0, b_{13}^{3}=0 .
\end{aligned}
$$

Thus,

$$
\begin{aligned}
& b(z, w)=\left(0, b_{21}^{2} z_{2} w_{1}+b_{22}^{2} z_{2} w_{2}+b_{23}^{2} z_{2} w_{3},\right. \\
& \left.b_{21}^{3} z_{2} w_{1}+b_{22}^{3} z_{2} w_{2}+b_{23}^{3} z_{2} w_{3}\right)
\end{aligned}
$$

and

$$
\begin{aligned}
B_{\mathbf{x}}(w)=\frac{1}{2}\left(0, b_{21}^{2} \mathbf{x}_{2} w_{1}+b_{22}^{2} \mathbf{x}_{2} w_{2}+b_{23}^{2} \mathbf{x}_{2} w_{3},\right. \\
\left.b_{21}^{3} \mathbf{x}_{2} w_{1}+b_{22}^{3} \mathbf{x}_{2} w_{2}+b_{23}^{3} \mathbf{x}_{2} w_{3}\right) .
\end{aligned}
$$

We will now utilize the requirement that $B_{\mathbf{x}}$ is associated to $A_{\mathbf{x}}$ with respect to $\mathcal{H}$ for every $\mathbf{x} \in \mathbb{R}^{2}$ as in (i) in Theorem 2.5. On the one hand, from (A.3) we have

$$
A_{\mathbf{x}} \mathcal{H}\left(w, w^{\prime}\right)=\left(a_{11}^{1} \mathbf{x}_{1} \alpha \bar{w}_{1} w_{1}^{\prime}, a_{22}^{2} \mathbf{x}_{2}\left(\gamma \bar{w}_{1} w_{1}^{\prime}+\delta \bar{w}_{2} w_{2}^{\prime}+\delta \bar{w}_{3} w_{3}^{\prime}\right)\right) .
$$

On the other hand, from (A.14) one obtains

$$
\begin{array}{r}
\mathcal{H}\left(B_{\mathbf{x}}(w), w^{\prime}\right)+\mathcal{H}\left(w, B_{\mathbf{x}}\left(w^{\prime}\right)\right)=\frac{1}{2}\left(0, \delta\left(\bar{b}_{21}^{2} \mathbf{x}_{2} \bar{w}_{1}+\bar{b}_{22}^{2} \mathbf{x}_{2} \bar{w}_{2}+\right.\right. \\
\left.\bar{b}_{23}^{2} \mathbf{x}_{2} \bar{w}_{3}\right) w_{2}^{\prime}+\delta\left(\bar{b}_{21}^{3} \mathbf{x}_{2} \bar{w}_{1}+\bar{b}_{22}^{3} \mathbf{x}_{2} \bar{w}_{2}+\bar{b}_{23}^{3} \mathbf{x}_{2} \bar{w}_{3}\right) w_{3}^{\prime}+\delta \bar{w}_{2}\left(b_{21}^{2} \mathbf{x}_{2} w_{1}^{\prime}+\right. \\
\left.\left.b_{22}^{2} \mathbf{x}_{2} w_{2}^{\prime}+b_{23}^{2} \mathbf{x}_{2} w_{3}^{\prime}\right)+\delta \bar{w}_{3}\left(b_{21}^{3} \mathbf{x}_{2} w_{1}^{\prime}+b_{22}^{3} \mathbf{x}_{2} w_{2}^{\prime}+b_{23}^{3} \mathbf{x}_{2} w_{3}^{\prime}\right)\right) .
\end{array}
$$

Comparing (A.15) and (A.16), we deduce

$$
a_{11}^{1}=0, a_{22}^{2}=0, b_{21}^{2}=0, b_{21}^{3}=0,
$$

in particular, $a=0$. 
By (A.17), formula (A.13) simplifies as

$$
b(z, w)=\left(0, b_{22}^{2} z_{2} w_{2}+b_{23}^{2} z_{2} w_{3}, b_{22}^{3} z_{2} w_{2}+b_{23}^{3} z_{2} w_{3}\right),
$$

and we will now use condition (iii) in Theorem 2.5. We have

$$
\begin{array}{r}
b\left(\mathcal{H}\left(w^{\prime}, w^{\prime \prime}\right), w^{\prime \prime}\right)=\left(0,\left(\gamma \bar{w}_{1}^{\prime} w_{1}^{\prime \prime}+\delta \bar{w}_{2}^{\prime} w_{2}^{\prime \prime}+\delta \bar{w}_{3}^{\prime} w_{3}^{\prime \prime}\right)\left(b_{22}^{2} w_{2}^{\prime \prime}+b_{23}^{2} w_{3}^{\prime \prime}\right),\right. \\
\left.\left(\gamma \bar{w}_{1}^{\prime} w_{1}^{\prime \prime}+\delta \bar{w}_{2}^{\prime} w_{2}^{\prime \prime}+\delta \bar{w}_{3}^{\prime} w_{3}^{\prime \prime}\right)\left(b_{22}^{3} w_{2}^{\prime \prime}+b_{23}^{3} w_{3}^{\prime \prime}\right)\right),
\end{array}
$$

therefore

$$
\begin{aligned}
& \mathcal{H}\left(w, b\left(\mathcal{H}\left(w^{\prime}, w^{\prime \prime}\right), w^{\prime \prime}\right)\right)=\left(0, \delta \bar{w}_{2}\left(\gamma \bar{w}_{1}^{\prime} w_{1}^{\prime \prime}+\delta \bar{w}_{2}^{\prime} w_{2}^{\prime \prime}+\delta \bar{w}_{3}^{\prime} w_{3}^{\prime \prime}\right) \times\right. \\
& \left.\left(b_{22}^{2} w_{2}^{\prime \prime}+b_{23}^{2} w_{3}^{\prime \prime}\right)+\delta \bar{w}_{3}\left(\gamma \bar{w}_{1}^{\prime} w_{1}^{\prime \prime}+\delta \bar{w}_{2}^{\prime} w_{2}^{\prime \prime}+\delta \bar{w}_{3}^{\prime} w_{3}^{\prime \prime}\right)\left(b_{22}^{3} w_{2}^{\prime \prime}+b_{23}^{3} w_{3}^{\prime \prime}\right)\right) .
\end{aligned}
$$

On the other hand

$$
\begin{array}{r}
b\left(\mathcal{H}\left(w^{\prime \prime}, w\right), w^{\prime}\right)=\left(0,\left(\gamma \bar{w}_{1}^{\prime \prime} w_{1}+\delta \bar{w}_{2}^{\prime \prime} w_{2}+\delta \bar{w}_{3}^{\prime \prime} w_{3}\right)\left(b_{22}^{2} w_{2}^{\prime}+b_{23}^{2} w_{3}^{\prime}\right),\right. \\
\left.\left(\gamma \bar{w}_{1}^{\prime \prime} w_{1}+\delta \bar{w}_{2}^{\prime \prime} w_{2}+\delta \bar{w}_{3}^{\prime \prime} w_{3}\right)\left(b_{22}^{3} w_{2}^{\prime}+b_{23}^{3} w_{3}^{\prime}\right)\right),
\end{array}
$$

hence

$$
\begin{array}{r}
\mathcal{H}\left(b\left(\mathcal{H}\left(w^{\prime \prime}, w\right), w^{\prime}\right), w^{\prime \prime}\right)=\left(0, \delta\left(\gamma w_{1}^{\prime \prime} \bar{w}_{1}+\delta w_{2}^{\prime \prime} \bar{w}_{2}+\delta w_{3}^{\prime \prime} \bar{w}_{3}\right) \times\right. \\
\left(\bar{b}_{22}^{2} \bar{w}_{2}^{\prime}+\bar{b}_{23}^{2} \bar{w}_{3}^{\prime}\right) w_{2}^{\prime \prime}+\delta\left(\gamma w_{1}^{\prime \prime} \bar{w}_{1}+\delta w_{2}^{\prime \prime} \bar{w}_{2}+\delta w_{3}^{\prime \prime} \bar{w}_{3}\right) \times \\
\left.\left(\bar{b}_{22}^{3} \bar{w}_{2}^{\prime}+\bar{b}_{23}^{3} \bar{w}_{3}^{\prime}\right) w_{3}^{\prime \prime}\right) .
\end{array}
$$

Comparing (A.18) and (A.19) we see that $b_{22}^{2}=0, b_{23}^{2}=0, b_{22}^{3}=0, b_{23}^{3}=0$ and therefore $b=0$. This proves that $\mathfrak{g}_{1}=0$ as claimed.

Suppose now that $\gamma=0$, hence each of $\beta, \delta$ is non-zero. By (A.12) we then have

$$
\begin{aligned}
& b_{21}^{1}=0, b_{22}^{1}=0, b_{23}^{1}=0, b_{11}^{2}=0, b_{12}^{2}=0, b_{13}^{2}=0, b_{21}^{2}=0, b_{22}^{2}=0, \\
& b_{23}^{2}=0, b_{11}^{3}=0, b_{12}^{3}=0, b_{13}^{3}=0, b_{21}^{3}=0, b_{22}^{3}=0, b_{23}^{3}=0,
\end{aligned}
$$

and (A.11) implies

$$
\operatorname{Im} b_{11}^{1}=0 .
$$

Thus,

$$
B_{\mathbf{x}}(w)=\frac{1}{2}\left(b_{11}^{1} \mathbf{x}_{1} w_{1}+b_{12}^{1} \mathbf{x}_{1} w_{2}+b_{13}^{1} \mathbf{x}_{1} w_{3}, 0,0\right) .
$$

We will now recall that $B_{\mathbf{x}}$ is associated to $A_{\mathbf{x}}$ with respect to $\mathcal{H}$ for every $\mathbf{x} \in \mathbb{R}^{2}$. On the one hand, from (A.3) we have

(A.22) $A_{\mathbf{x}} \mathcal{H}\left(w, w^{\prime}\right)=\left(a_{11}^{1} \mathbf{x}_{1}\left(\alpha \bar{w}_{1} w_{1}^{\prime}+\beta \bar{w}_{2} w_{2}^{\prime}+\beta \bar{w}_{3} w_{3}^{\prime}\right), a_{22}^{2} \mathbf{x}_{2}\left(\delta \bar{w}_{2} w_{2}^{\prime}+\delta \bar{w}_{3} w_{3}^{\prime}\right)\right)$.

On the other hand, from (A.21) one obtains

$$
\begin{aligned}
\mathcal{H}\left(B_{\mathbf{x}}(w), w^{\prime}\right)+\mathcal{H}\left(w, B_{\mathbf{x}}\left(w^{\prime}\right)\right)=\frac{1}{2}\left(\alpha \left(\bar{b}_{11}^{1} \mathbf{x}_{1} \bar{w}_{1}+\bar{b}_{12}^{1} \mathbf{x}_{1} \bar{w}_{2}+\right.\right. \\
\left.\left.\bar{b}_{13}^{1} \mathbf{x}_{1} \bar{w}_{3}\right) w_{1}^{\prime}+\alpha \bar{w}_{1}\left(b_{11}^{1} \mathbf{x}_{1} w_{1}^{\prime}+b_{12}^{1} \mathbf{x}_{1} w_{2}^{\prime}+b_{13}^{1} \mathbf{x}_{1} w_{3}^{\prime}\right), 0\right) .
\end{aligned}
$$

Comparing (A.22) and (A.23), we see

$$
a_{11}^{1}=0, a_{22}^{2}=0, \operatorname{Re} b_{11}^{1}=0, b_{12}^{1}=0, b_{13}^{1}=0 .
$$

By (A.20), (A.24) it follows that $a=0, b=0$, which shows that $\mathfrak{g}_{1}=0$.

To complete the proof, notice that the case $\delta=0$ is obtained from the case $\beta=0$ by permutation of variables.

Finally, we consider the domain $D_{6}$.

Proposition A.10. For $\mathfrak{g}=\mathfrak{g}\left(D_{6}\right)$ one has $\operatorname{dim} \mathfrak{g}_{1}=1$. 
Proof. As in the proof of Lemma 3.6, we assume that $v=(1,1,0)$. We will utilize Theorem 2.5 for the cone $\Omega_{3}$ and the $\Omega_{3}$-Hermitian form $\mathcal{H}$ defined in (3.21).

Let $b: \mathbb{C}^{3} \times \mathbb{C} \rightarrow \mathbb{C}$ be a $\mathbb{C}$-bilinear map:

$$
b(z, w)=\left(b_{1} z_{1}+b_{2} z_{2}+b_{3} z_{3}\right) w,
$$

where $b_{j} \in \mathbb{C}$. For every fixed pair $\mathbf{w}, \mathbf{w}^{\prime} \in \mathbb{C}$ we compute

$$
\mathcal{H}\left(\mathbf{w}^{\prime}, b(x, \mathbf{w})\right)=\left(\overline{\mathbf{w}}^{\prime} \mathbf{w}\left(b_{1} x_{1}+b_{2} x_{2}+b_{3} x_{3}\right), \overline{\mathbf{w}}^{\prime} \mathbf{w}\left(b_{1} x_{1}+b_{2} x_{2}+b_{3} x_{3}\right), 0\right),
$$

with $x \in \mathbb{R}^{3}$. Then from (ii) of Theorem 2.5 we obtain

$$
\begin{aligned}
B_{\mathbf{w}, \mathbf{w}^{\prime}}(x)=\left(\operatorname{Im}\left(b_{1} \overline{\mathbf{w}}^{\prime} \mathbf{w}\right) x_{1}+\operatorname{Im}\left(b_{2} \overline{\mathbf{w}}^{\prime} \mathbf{w}\right) x_{2}+\operatorname{Im}\left(b_{3} \overline{\mathbf{w}}^{\prime} \mathbf{w}\right) x_{3},\right. \\
\left.\operatorname{Im}\left(b_{1} \overline{\mathbf{w}}^{\prime} \mathbf{w}\right) x_{1}+\operatorname{Im}\left(b_{2} \overline{\mathbf{w}}^{\prime} \mathbf{w}\right) x_{2}+\operatorname{Im}\left(b_{3} \overline{\mathbf{w}}^{\prime} \mathbf{w}\right) x_{3}, 0\right) .
\end{aligned}
$$

By (3.22), the condition that this map lies in $\mathfrak{g}\left(\Omega_{3}\right)$ for all $\mathbf{w}, \mathbf{w}^{\prime} \in \mathbb{C}$ immediately yields

$$
\operatorname{Im}\left(b_{1} \overline{\mathbf{w}}^{\prime} \mathbf{w}\right) \equiv 0, \quad \operatorname{Im}\left(b_{2} \overline{\mathbf{w}}^{\prime} \mathbf{w}\right) \equiv 0, \quad \operatorname{Im}\left(b_{3} \overline{\mathbf{w}}^{\prime} \mathbf{w}\right) \equiv 0,
$$

hence $b=0$.

Next, consider a symmetric $\mathbb{R}$-bilinear form on $\mathbb{R}^{3}$ with values in $\mathbb{R}^{3}$ :

$$
\begin{aligned}
a(x, x)= & \left(a_{11}^{1} x_{1}^{2}+2 a_{12}^{1} x_{1} x_{2}+2 a_{13}^{1} x_{1} x_{3}+a_{22}^{1} x_{2}^{2}+2 a_{23}^{1} x_{2} x_{3}+a_{33}^{1} x_{3}^{2},\right. \\
& a_{11}^{2} x_{1}^{2}+2 a_{12}^{2} x_{1} x_{2}+2 a_{13}^{2} x_{1} x_{3}+a_{22}^{2} x_{2}^{2}+2 a_{23}^{2} x_{2} x_{3}+a_{33}^{2} x_{3}^{2}, \\
& \left.a_{11}^{3} x_{1}^{2}+2 a_{12}^{3} x_{1} x_{2}+2 a_{13}^{3} x_{1} x_{3}+a_{22}^{3} x_{2}^{2}+2 a_{23}^{3} x_{2} x_{3}+a_{33}^{3} x_{3}^{2}\right),
\end{aligned}
$$

where $a_{i j}^{k} \in \mathbb{R}$. Then for a fixed $\mathbf{x} \in \mathbb{R}^{3}$ from (2.14) we compute

$$
\begin{aligned}
& A_{\mathbf{x}}(x)=\left(a_{11}^{1} \mathbf{x}_{1} x_{1}+a_{12}^{1} \mathbf{x}_{1} x_{2}+a_{12}^{1} \mathbf{x}_{2} x_{1}+a_{13}^{1} \mathbf{x}_{1} x_{3}+a_{13}^{1} \mathbf{x}_{3} x_{1}+a_{22}^{1} \mathbf{x}_{2} x_{2}+\right. \\
& a_{23}^{1} \mathbf{x}_{2} x_{3}+a_{23}^{1} \mathbf{x}_{3} x_{2}+a_{33}^{1} \mathbf{x}_{3} x_{3}, a_{11}^{2} \mathbf{x}_{1} x_{1}+a_{12}^{2} \mathbf{x}_{1} x_{2}+a_{12}^{2} \mathbf{x}_{2} x_{1}+a_{13}^{2} \mathbf{x}_{1} x_{3}+ \\
& a_{13}^{2} \mathbf{x}_{3} x_{1}+a_{22}^{2} \mathbf{x}_{2} x_{2}+a_{23}^{2} \mathbf{x}_{2} x_{3}+a_{23}^{2} \mathbf{x}_{3} x_{2}+a_{33}^{2} \mathbf{x}_{3} x_{3}, a_{11}^{3} \mathbf{x}_{1} x_{1}+a_{12}^{3} \mathbf{x}_{1} x_{2}+ \\
&\left.a_{12}^{3} \mathbf{x}_{2} x_{1}+a_{13}^{3} \mathbf{x}_{1} x_{3}+a_{13}^{3} \mathbf{x}_{3} x_{1}+a_{22}^{3} \mathbf{x}_{2} x_{2}+a_{23}^{3} \mathbf{x}_{2} x_{3}+a_{23}^{3} \mathbf{x}_{3} x_{2}+a_{33}^{3} \mathbf{x}_{3} x_{3}\right)= \\
&\left(\left(a_{11}^{1} \mathbf{x}_{1}+a_{12}^{1} \mathbf{x}_{2}+a_{13}^{1} \mathbf{x}_{3}\right) x_{1}+\left(a_{12}^{1} \mathbf{x}_{1}+a_{22}^{1} \mathbf{x}_{2}+a_{23}^{1} \mathbf{x}_{3}\right) x_{2}+\left(a_{13}^{1} \mathbf{x}_{1}+a_{23}^{1} \mathbf{x}_{2}+\right.\right. \\
&\left.a_{33}^{1} \mathbf{x}_{3}\right) x_{3},\left(a_{11}^{2} \mathbf{x}_{1}+a_{12}^{2} \mathbf{x}_{2}+a_{13}^{2} \mathbf{x}_{3}\right) x_{1}+\left(a_{12}^{2} \mathbf{x}_{1}+a_{22}^{2} \mathbf{x}_{2}+a_{23}^{2} \mathbf{x}_{3}\right) x_{2}+\left(a_{13}^{2} \mathbf{x}_{1}+\right. \\
&\left.a_{23}^{2} \mathbf{x}_{2}+a_{33}^{2} \mathbf{x}_{3}\right) x_{3},\left(a_{11}^{3} \mathbf{x}_{1}+a_{12}^{3} \mathbf{x}_{2}+a_{13}^{3} \mathbf{x}_{3}\right) x_{1}+\left(a_{12}^{3} \mathbf{x}_{1}+a_{22}^{3} \mathbf{x}_{2}+a_{23}^{3} \mathbf{x}_{3}\right) x_{2}+ \\
&\left.\left(a_{13}^{3} \mathbf{x}_{1}+a_{23}^{3} \mathbf{x}_{2}+a_{33}^{3} \mathbf{x}_{3}\right) x_{3}\right)
\end{aligned}
$$

where $x \in \mathbb{R}^{3}$. By (3.22), the condition that this map lies in $\mathfrak{g}\left(\Omega_{3}\right)$ for every $\mathbf{x} \in \mathbb{R}^{3}$ is equivalent to

$$
\begin{aligned}
& a_{11}^{1} \mathbf{x}_{1}+a_{12}^{1} \mathbf{x}_{2}+a_{13}^{1} \mathbf{x}_{3} \equiv a_{12}^{2} \mathbf{x}_{1}+a_{22}^{2} \mathbf{x}_{2}+a_{23}^{2} \mathbf{x}_{3} \equiv \\
& a_{13}^{3} \mathbf{x}_{1}+a_{23}^{3} \mathbf{x}_{2}+a_{33}^{3} \mathbf{x}_{3} \\
& a_{12}^{1} \mathbf{x}_{1}+a_{22}^{1} \mathbf{x}_{2}+a_{23}^{1} \mathbf{x}_{3} \equiv a_{11}^{2} \mathbf{x}_{1}+a_{12}^{2} \mathbf{x}_{2}+a_{13}^{2} \mathbf{x}_{3} \\
& a_{13}^{1} \mathbf{x}_{1}+a_{23}^{1} \mathbf{x}_{2}+a_{33}^{1} \mathbf{x}_{3} \equiv a_{11}^{3} \mathbf{x}_{1}+a_{12}^{3} \mathbf{x}_{2}+a_{13}^{3} \mathbf{x}_{3} \\
& a_{13}^{2} \mathbf{x}_{1}+a_{23}^{2} \mathbf{x}_{2}+a_{33}^{2} \mathbf{x}_{3} \equiv-\left(a_{12}^{3} \mathbf{x}_{1}+a_{22}^{3} \mathbf{x}_{2}+a_{23}^{3} \mathbf{x}_{3}\right)
\end{aligned}
$$

Further, recalling that any map $b: \mathbb{C}^{3} \times \mathbb{C} \rightarrow \mathbb{C}$ as above is zero, we will utilize the condition that the zero matrix is associated to $A_{\mathbf{x}}$ for every $\mathbf{x} \in \mathbb{R}^{3}$ as in (i) of Theorem 2.5. This condition means

$$
\begin{aligned}
& a_{12}^{1} \mathbf{x}_{1}+a_{22}^{1} \mathbf{x}_{2}+a_{23}^{1} \mathbf{x}_{3} \equiv-\left(a_{11}^{1} \mathbf{x}_{1}+a_{12}^{1} \mathbf{x}_{2}+a_{13}^{1} \mathbf{x}_{3}\right) \\
& a_{13}^{2} \mathbf{x}_{1}+a_{23}^{2} \mathbf{x}_{2}+a_{33}^{2} \mathbf{x}_{3} \equiv a_{11}^{3} \mathbf{x}_{1}+a_{12}^{3} \mathbf{x}_{2}+a_{13}^{3} \mathbf{x}_{3}
\end{aligned}
$$


Combining identities (A.25) and (A.26), we obtain the following relations for the coefficients of the form $a$ :

$$
\begin{aligned}
& a_{11}^{1}=a_{12}^{2}=a_{13}^{3}, a_{12}^{1}=a_{22}^{2}=a_{23}^{3}, a_{13}^{1}=a_{23}^{2}=a_{33}^{3}, a_{12}^{1}=a_{11}^{2}, a_{22}^{1}=a_{12}^{2}, a_{23}^{1}=a_{13}^{2}, \\
& a_{13}^{1}=a_{11}^{3}, a_{23}^{1}=a_{12}^{3}, a_{33}^{1}=a_{13}^{3}, a_{13}^{2}=-a_{12}^{3}, a_{23}^{2}=-a_{22}^{3}, a_{33}^{2}=-a_{23}^{3}, a_{12}^{1}=-a_{11}^{1}, \\
& a_{22}^{1}=-a_{12}^{1}, a_{23}^{1}=-a_{13}^{1}, a_{13}^{2}=a_{11}^{3}, a_{23}^{2}=a_{12}^{3}, a_{33}^{2}=a_{13}^{3} .
\end{aligned}
$$

By the above relations, each coefficient of $a$ either is zero or is equal to $\pm a_{11}^{1}$ as follows:

$$
\begin{aligned}
& a_{12}^{1}=-a_{11}^{1}, a_{13}^{1}=0, a_{22}^{1}=a_{11}^{1}, a_{23}^{1}=0, a_{33}^{1}=a_{11}^{1}, \\
& a_{11}^{2}=-a_{11}^{1}, a_{12}^{2}=a_{11}^{1}, a_{13}^{2}=0, a_{22}^{2}=-a_{11}^{1}, a_{23}^{2}=0, \\
& a_{33}^{2}=a_{11}^{1}, a_{11}^{3}=0, a_{12}^{3}=0, a_{13}^{3}=a_{11}^{1}, a_{22}^{3}=0, a_{23}^{3}=-a_{11}^{1}, a_{33}^{3}=0 .
\end{aligned}
$$

Therefore

$$
a(x, x)=a_{11}^{1}\left(\left(x_{1}-x_{2}\right)^{2}+x_{3}^{2},-\left(x_{1}-x_{2}\right)^{2}+x_{3}^{2}, 2\left(x_{1}-x_{2}\right) x_{3}\right) .
$$

This shows that $\operatorname{dim} \mathfrak{g}_{1}=1$ as required.

\section{REFERENCES}

[C] Cartan, É., Sur les domaines bornés homogènes de l'espace de $n$ variables complexes, Abh. Math. Sem. Univ. Hamburg 11 (1935), 116-162.

[D] Dorfmeister, J., Homogeneous Siegel domains, Nagoya Math. J. 86 (1982), 39-83.

[GIL] Geatti, L., Iannuzzi, A. and Loeb, J.-J., Hyperbolic manifolds whose envelopes of holomorphy are not hyperbolic, https://arxiv.org/abs/math/0612182.

[I1] Isaev, A. V., Hyperbolic manifolds of dimension $n$ with automorphism group of dimension $n^{2}-1$, J. Geom. Anal. 15 (2005), 239-259.

[I2] Isaev, A. V., Hyperbolic $n$-dimensional manifolds with automorphism group of dimension $n^{2}$, Geom. Funct. Anal. (GAFA) 17 (2007), 192-219.

[I3] Isaev, A. V., Lectures on the Automorphism Groups of Kobayashi-Hyperbolic Manifolds, Lecture Notes in Mathematics, 1902, Springer, Berlin, 2007.

[I4] Isaev, A. V., Hyperbolic 2-dimensional manifolds with 3-dimensional automorphism group, Geom. Topol. 12 (2008), 643-711.

[I5] Isaev, A. V., Proper actions of high-dimensional groups on complex manifolds, Bull. Math. Sci. 5 (2015), 251-285.

[IK] Isaev, A. V. and Krantz, S. G., On the automorphism groups of hyperbolic manifolds, J. reine angew. Math. $\mathbf{5 3 4}$ (2001), 187-194.

[KT] Kaneyuki, S. and Tsuji, T., Classification of homogeneous bounded domains of lower dimension, Nagoya Math. J. 53 (1974), 1-46.

[KMO] Kaup, W., Matsushima, Y. and Ochiai, T., On the automorphisms and equivalences of generalized Siegel domains, Amer. J. Math. 92 (1970), 475-497.

[K1] Kobayashi, S., Hyperbolic Manifolds and Holomorphic Mappings, Marcel Dekker, New York, 1970

[K2] Kobayashi, S., Hyperbolic Complex Spaces, Grundlehren der Mathematischen Wissenschaften, 318, Springer-Verlag, Berlin, 1998.

[N] Nakajima, K., Homogeneous hyperbolic manifolds and homogeneous Siegel domains, J. Math. Kyoto Univ. 25 (1985), 269-291.

[P-S] Pyatetskii-Shapiro, I. I., Automorphic Functions and the Geometry of Classical Domains, Gordon and Breach, New York, 1969.

[R] Rudin, W., Function Theory in the Unit Ball of $\mathbb{C}^{n}$, Grundlehren der Mathematischen Wissenschaften, 241, Springer, New York-Berlin, 1980.

[S] Satake, I., Algebraic Structures of Symmetric Domains, Kanô Memorial Lectures, 4, Princeton University Press, 1980.

[V] Verma, K., A characterization of domains in $\mathbb{C}^{2}$ with noncompact automorphism group, Math. Ann. 344 (2009), 645-701.

[VGP-S] Vinberg, E. B., Gindikin, S. G. and Pjateckiı̌-Šapiro, I. I., Classification and canonical realization of complex bounded homogeneous domains, Trans. Mosc. Math. Soc. 12 (1963), 404-437. 
Mathematical Sciences Institute, Australian National University, Canberra, Acton, ACT 2601, Australia

E-mail address: alexander.isaev@anu.edu.au 